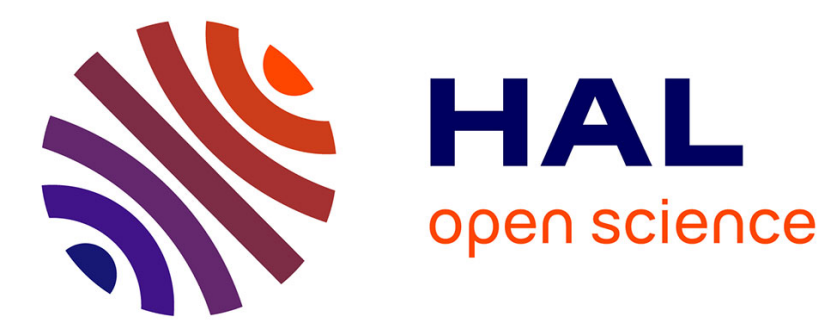

\title{
Instability in the Transformation between Extensional and Flexural Modes in Thin-Walled Cylindrical Shells
}

\author{
M.X. Shi, Q.M. Li
}

\section{To cite this version:}

M.X. Shi, Q.M. Li. Instability in the Transformation between Extensional and Flexural Modes in Thin-Walled Cylindrical Shells. European Journal of Mechanics - A/Solids, 2010, 30 (1), pp.33. 10.1016/j.euromechsol.2010.09.002 . hal-00696139

\section{HAL Id: hal-00696139 \\ https://hal.science/hal-00696139}

Submitted on 11 May 2012

HAL is a multi-disciplinary open access archive for the deposit and dissemination of scientific research documents, whether they are published or not. The documents may come from teaching and research institutions in France or abroad, or from public or private research centers.
L'archive ouverte pluridisciplinaire HAL, est destinée au dépôt et à la diffusion de documents scientifiques de niveau recherche, publiés ou non, émanant des établissements d'enseignement et de recherche français ou étrangers, des laboratoires publics ou privés. 


\section{Accepted Manuscript}

Title: Instability in the Transformation between Extensional and Flexural Modes in

Thin-Walled Cylindrical Shells

Authors: M.X. Shi, Q.M. Li

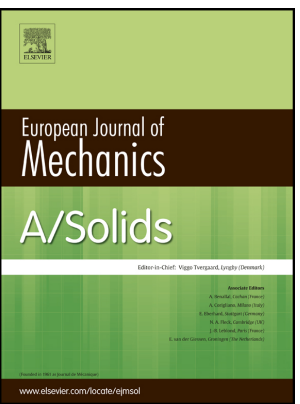

PII: S0997-7538(10)00102-6

DOI: $\quad$ 10.1016/j.euromechsol.2010.09.002

Reference: EJMSOL 2637

To appear in: European Journal of Mechanics / A Solids

Received Date: 1 May 2009

Revised Date: 2 September 2010

Accepted Date: 3 September 2010

Please cite this article as: Shi, M.X., Li, Q.M. Instability in the Transformation between Extensional and Flexural Modes in Thin-Walled Cylindrical Shells, European Journal of Mechanics / A Solids (2010), doi: 10.1016/j.euromechsol.2010.09.002

This is a PDF file of an unedited manuscript that has been accepted for publication. As a service to our customers we are providing this early version of the manuscript. The manuscript will undergo copyediting, typesetting, and review of the resulting proof before it is published in its final form. Please note that during the production process errors may be discovered which could affect the content, and all legal disclaimers that apply to the journal pertain. 
Instability in the Transformation between Extensional and Flexural Modes in

Thin-Walled Cylindrical Shells

\author{
M.X. Shi ${ }^{1,2}$, Q.M. Li $^{1, a}$ \\ ${ }^{1}$ School of Mechanical, Aerospace and Civil Engineering, Pariser Building, \\ The University of Manchester, PO BOX 88, Manchester M60 1QD, UK \\ ${ }^{2}$ Department of Engineering Mechanics, College of Science, \\ Chang'an University, Xi' an 710064, China
}

\begin{abstract}
With the inclusion of all terms up to the third-order in the membrane strain to consider the geometric nonlinearity in deformation, a third-order implicit model and a fourth-order explicit model for the vibration transformation between extensional and flexural modes in thin-walled cylindrical shells are established and solved numerically. Numerical instability is observed in numerical solutions based on the explicit model. It is found that such numerical instability does not result from the accumulated numerical errors in the numerical integration process, but from the neglecting of higher order terms in the formulation of the problem. With the inclusion of all terms up to the fourth-order in the strain energy, the explicit model can predict a stable vibration history with periodic mode transformations between the two modes. The same 2:1 internal resonance of the vibration mode transformation is predicted by both models. As flexural stress growth is concerned, the two models matches very well during the first group of peaks but lag in phase gradually appears in later group of stress peaks based on the implicit model. This is understood to be resulting from the limited number of terms included in the series expansions based on the explicit model, which allows the most likely excited flexural mode get a larger share of energy transferred from the principle mode and as a result flexural stress arrives at peaks earlier based on the explicit model.
\end{abstract}

Keywords: thin-walled shell, extensional mode, flexural mode, mode transformation, numerical instability

${ }^{a}$ Corresponding author: Qingming.Li@manchester.ac.uk 


\section{Introduction}

The investigation on nonlinear dynamics of circular cylindrical shells has received considerable attentions since the middle of fifties of last century. The nonlinearity may come from sources such as geometry, material, inertia or any combination of them. The geometric nonlinearity stems either from the initial boundary conditions or nonlinear strain- and/or curvature-displacement relations. The material nonlinearity occurs when the stresses are non-linear functions of strains, or vice versa. The inertia nonlinearity may be caused by the presence of non-uniform mass distribution. The nonlinearity of the problem eventually appears either in the governing partial differential equations and/or in the boundary conditions. Most of the present studies deal with geometric nonlinearities and the main differences among them are the approximations used in establishing strain and/or curvature relations with displacements. A series of reviews on nonlinear dynamics of shell structures in past decades have been published with emphasis on different subjects of the topic (Evensen, 1974, Leissa, 1984, Qatu, 1992, Liew et al., 1997, Kubenko and Koval'chuk, 1998, Moussaoui and Benamar, 2002, Amabili and Paidoussis, 2003, Krysko et al., 2008). Besides, effects of transverse deformation and rotary inertia for moderately thick shells were reviewed in reference (Liew et al., 1997). An extensive review with 356 references listed in chronological order on the subject of dynamics of circular cylindrical shells was given in reference (Amabili and Paidoussis, 2003).

The pioneering works in (Grigolyuk, 1955a,1955b, Reissner, 1955a,1955b) established the nonlinear partial differential equations governing vibrations of cylindrical and spherical shells with finite deflections. Mode expansion method in the nonlinear dynamic analysis of rings and cylindrical shells was introduced in (Evensen, 1963, Evensen, 1964, Evensen, 1967), which strongly supported earlier experimental observations of nonlinear effects of large amplitude on vibration frequencies in finite length circular cylindrical shells. Some classic theoretical and experimental developments on nonlinear dynamics of circular rings and cylindrical shells were also published in (Goodier and Mcivor, 1964, Evensen, 1966, Mcivor, 1966, Dowell, 1967, Dowell and Ventres, 1968, Mcivor and Lovell, 1968, Evensen, 1974, Lindberg, 1974). On one hand, the well-known Donnell shallow shell theory (Donnell, 1938) were employed to study the amplitude-frequency characteristics (AFCs) of finite length cylindrical shells (Evensen, 1966, 


\section{ACCEPTED MANUSCRIPT}

Dowell, 1967, Evensen, 1967, Dowell and Ventres, 1968), which has continuously attracted attentions from scientists (Dowell, 1998, Amabili et al., 1999, Evensen, 1999). On the other hand, in McIvor and his colleagues' publications on rings and circular cylindrical shells (Goodier and McIvor, 1964, McIvor, 1966, Lovell and McIvor, 1969), vibration features of rings and circular cylindrical shells under nearly uniform radial impulse were characterized by a parametric excitation of axisymmetric and/or asymmetric modes. By means of Lagrange equation, they reduced the ordinary-differential equations to a series of nonlinear equations revealing the coupling between the radial breathing mode and flexural modes. Further linearization of the coupled non-linear differential equations led to a Mathieu-type equation, which indicates the stable and unstable regions of the problem and demonstrates the 2:1 internal resonance mechanism when the most likely mode transformations can be initiated from the first unstable region of Mathieu stability diagram (Mclachilan, 1947, Struble, 1962). Intermittent mode transformations could be numerically predicted for circular cylindrical shells under certain circumstances and the earliest study on this topic should be credited to Goodier and his colleagues for their pioneering work (Goodier and McIvor, 1964, McIvor, 1966). Later Nayfeh and Raouf used multiple scale method to get a perturbation solution of the same problem (Nayfeh and Raouf, 1987). The perturbation approach is effective in delineating mode interactions when frequencies of several successive modes are closely grouped together. In their work, the phenomenon of energy saturation of the driven mode (radial breathing mode) was observed and it was also predicted that the mode transformations might not be steady-state periodic. Instead, cylindrical shells might experience amplitude- and phase-modulated chaotic vibrations. In the case of simply supported circular cylindrical shells filled with a quiescent fluid, it is shown that the coupling between driven mode and excited modes is always linked nonlinearly in the normal form of the system and an internal resonance 1:1:1:2 phenomenon was observed by Amabili and his colleagues using an analytic approach, i.e. the method of normal mode forms (Amabili et al., 2000). This problem was further investigated by a perturbation analysis (Pellicano et al., 2000) focusing on mode interactions and the same nonlinearly coupled vibrations and internal resonance phenomena were found. For thin-walled spherical shell structures, extensive studies have also been carried out with focus analogously on the intrinsic nonlinearities and internal resonance characterizing vibrations (McIvor and Sonstegard, 1966, Ye, 1997, Nayfeh, 2000, Thomas et al., 2005, Nayfeh and Arafat, 2006, Touze and Thomas, 2006, Thomas et al., 2007, 
Camier et al., 2009).

Apart from the previously mentioned classical shell theory, i.e. the Donnell shell theory or the similar theory under other names (Thomas et al., 2005), more refined thin shell theories have been proposed, such as (1) Sanders-Koiter theory (Sanders, 1959, Yamaki, 1984), (2)Flü gge-Lur'ye-Byrne theory (Flugge, 1934, Lur'ye, 1940, Byrne, 1944), and (3) Novozhilov's theory (Novozhilov, 1964). Among these thin shell theories, one common feature is that they all employed kinematic and mechanics hypotheses to describe the three-dimensional deformation of a representative shell element by deformation of the middle surface as a two-dimensional mechanics problem. Differences among them are the various assumptions made on the form of small terms included in strain and curvature expressions and the order of small terms retained in strain- and/or curvature-displacement relations. It may be necessary to point out that only linear terms are included in the curvature expressions in Donnell and Sander-Koiter's theories. Above four shell theories and an improved Novozhilov theory were evaluated in comparison with each other in a study of nonlinearity characteristics of thin circular cylindrical shells with large amplitude deformation (Amabili, 2003). For moderately thick circular cylindrical shells, a first-order shear deformation theory was established by Reddy and his colleagues (Reddy, 1984a,1984b, Reddy and Chandrashekhara, 1985a,1985b, Reddy and Liu, 1985), which is actually a thick-shell version of the Sanders-Koiter thin shell theory where five independent variables (including three displacements and two rotations) are used to describe the deformation. Only linear terms of the five deformation variables are included in the middle surface curvatures in this first-order moderately thick shell theory. The circumferential curvature is the main concern in the study of flexural deformation of circular cylindrical shells. In contrast to the assumed forms of small terms in previously mentioned four thin shell theories and completely linear terms in Reddy and his colleagues' thick shell theory, the derivation of curvature expression in Goodier and McIvor's theory is mathematically more rigorous (Goodier and McIvor, 1964, McIvor, 1966).

The radial "breathing" mode (RBM) in a cylindrical shell may be initiated by various means, e.g. when it is subjected to a nearly uniform radial impulse. Under certain conditions, strong flexural motion may be developed from unavoidable existence of imperfections in the radial impulse 
and/or geometric imprecations. Interesting intermittent transformations between RBM and flexural mode(s) may occur subsequently, accompanied with energy transfer between vibration modes (Goodier and McIvor, 1964, McIvor, 1966, McIvor and Lovell, 1968). Recently, it has been revealed that vibration mode transformation is a common phenomenon that may happen in engineering structures at very different length scales governed by $2: 1$ or similar internal resonance mechanism. For example, strain growth in explosion containment vessels is related to the vibration mode transformation (Zhu et al., 1997, Duffey and Romero, 2003, Li et al., 2008) and the intermittent mode transformation in single-walled carbon nanotubes was predicted in $(\mathrm{Li}$ and Shi, 2008, Shi et al., 2009a,2009b, Shi et al., 2009c). Since many natural, bio- and engineering structures have similar geometries to cylindrical and spherical shells, the vibration mode transformation may be realised in all these structures.

However, in our recent publications to study the intermittent transformation between RBM and flexural vibration modes in a single-walled carbon nanotube (Li and Shi, 2008), it was found that some discarded higher order terms in the formulation of problem (Goodier and McIvor, 1964, McIvor, 1966, McIvor and Lovell, 1968) may need to be retained in the strain energy expression to ensure stable vibration mode transformations, which will be studied in details in this paper with focusing on an infinitely long circular cylindrical shell, or thin-walled circular continuum ring, the same classic problem studied decades ago (Goodier and McIvor, 1964, McIvor, 1966). We will include all terms up to the fourth-order in the strain energy in this study such that the conservation of the total energy in the insulated mechanics system can be guaranteed. The basic formulation of the problem in terms of two non-dimensional variables is given in the first half part of section 2, which is called an implicit model for the problem. In the second half part of section 2 an explicit model is introduced by expanding the two variables into series and then using Lagrange equations to obtain the governing equations of the unknown coefficient functions of the series. Both models are a natural extension of the early formulation in (Goodier and McIvor, 1964). Section 3 presents the numerical results based on explicit model, focusing on the stability of vibration mode transformations and its dependence on the fourth-order term in strain energy. In section 4 the numerical results based on implicit model are given, focusing on flexural stress growth. The differences between explicit and implicit models are also presented in this section through one numerical example. Finally, main findings of this improved formulation of 
problem are summarized.

\section{Formulation of the problem}

\subsection{Implicit model for mode transformation}

When an infinitely long cylindrical shell undergoes deformation independent of axial coordinate, the original problem can be reduced to a two-dimensional plane strain problem. As shown in Fig.2.1, a point $P$ on the undeformed midsurface of a thin cylindrical shell of radius $a$ and thickness $h$ moves to point $P^{*} .(a, \theta)$ and $(r, \phi)$ are the polar coordinates of points $P$ and $P^{*}$, respectively, and the coordinates of the current position $P^{*}$ are functions of the coordinates of the original position $P$, i.e. $r=r(\theta, t)$ and $\phi=\phi(\theta, t)$. For a typical element (unit length in axial direction is taken for plane strain), as shown in Fig.2.2, its motion from the undeformed position (arc length $d s_{0}=a d \theta$ and curvature $\kappa_{0}=1 / a$ at initial stage, the right dashed-line element) to the current position (arc length $d s=\sqrt{d x^{2}+d y^{2}}=\sqrt{r^{\prime 2}+r^{2} \phi^{\prime 2}} d \theta$ and curvature $\kappa=d(\phi-\beta) / d s$ at current stage, the left solid-line element) is governed by Newton's second law in the normal and tangential directions of the deformed element (the current configuration), i.e.

$$
\begin{aligned}
& p(1+\varepsilon)+Q^{\prime} / a-\kappa(1+\varepsilon) S=\rho h\left(-a_{r} \cos \beta+a_{\theta} \sin \beta\right), \\
& S^{\prime} / a+\kappa(1+\varepsilon) Q=-\rho h\left(a_{r} \sin \beta+a_{\theta} \cos \beta\right)
\end{aligned}
$$

where the prime here and all thereafter indicates partial derivatives with respect to angle $\theta$. In Eqns.(2.1,2), $p$ is the pressure imposed on shell surface, $\rho$ the mass density, $S$ the normal force (compression positive), and $Q$ the shear force, respectively. $\varepsilon$ and $\kappa$ are the membrane strain and curvature at the point $(a, \theta)$, respectively, and will be defined later. $\beta$ is the angle between surface normal and the radius vector $\vec{r}$, which, in a sense of infinitesimal deformation, (since $d r$ and $r d \phi$ are the displacements, respectively, in the radial and tangent directions), can be determined by 
$\tan \beta=d r /(r d \phi)=r^{\prime} /\left(r \phi^{\prime}\right)$

When centroid of the cross-section is taken as fixed, acceleration components $a_{r}$ and $a_{\theta}$ of the element in Eqns.(2.1,2) are given as

$a_{r}=\ddot{r}-r \dot{\phi}^{2}, \quad a_{\theta}=r \ddot{\phi}+2 \dot{r} \dot{\phi}$,

where the dot above symbols refers to derivatives with respect to time $t$. When rotary inertia of the element is neglected, the moment equilibrium equation can lead to

$M^{\prime}+(1+\varepsilon) a Q=0$

Considering the relationship between moment and shear force given by Eqn.(2.5), the governing Eqns. $(2.1,2)$ can be reduced to a set of equations of normal force $S$ and moment $M$.

For the deformation shown in Fig.2.2, the membrane strain (engineering) and curvature at the point $(a, \theta)$ can be determined, respectively, by

$$
\begin{aligned}
& \varepsilon=\frac{d s-d s_{0}}{d s_{0}}=a^{-1} \sqrt{r^{\prime 2}+r^{2} \phi^{\prime 2}}-1, \\
& \kappa=\frac{d(\phi-\beta)}{d s}=\left[\phi^{\prime}\left(r^{2} \phi^{\prime 2}-r r^{\prime \prime}+2 r^{\prime 2}\right)+\phi^{\prime \prime} r r^{\prime}\right]\left(r^{\prime 2}+r^{2} \phi^{\prime 2}\right)^{-3 / 2}
\end{aligned}
$$

The relationship between angle $\beta$ and $(r, \phi)$ given by Eqn.(2.3) is used to derive the curvature in Eqn.(2.7). Under the assumption of small deformation, i.e. the surface normal and thickness of the shell wall remain unchanged during deformation, the shear deformation along thickness is ignored. Then, for a point at distance $z$ above middle surface, as shown in Fig.2.3, the strain $\varepsilon_{\theta}(z)$ and stress $\sigma_{\theta}(z)$ for plane strain can be obtained as

$$
\begin{aligned}
& \varepsilon_{\theta}(z)=\varepsilon+(1+\varepsilon)(a \kappa-1)\left(1-\frac{z}{a}\right) \frac{z}{a} \\
& \sigma_{\theta}(z)=E_{1} \varepsilon_{\theta}(z)
\end{aligned}
$$

in which the membrane stiffness $E_{1}$ and flexural stiffness $D$ are defined as 


$$
E_{1}=\frac{E}{1-v^{2}}, \quad D=\frac{E h^{3}}{12\left(1-v^{2}\right)}
$$

where $E$ and $v$ are Young's modulus and Poisson's ratio, respectively. Then the normal force $S$ and moment $M$ in Eqns.(2.1, 2,5) can be easily obtained based on the stress distribution along thickness given by Eqn.(2.9), i.e.

$$
\begin{aligned}
& S=-\int_{-h / 2}^{h / 2} \sigma_{\theta}(z) d z=-E_{1} h \varepsilon+D(1+\varepsilon)(a \kappa-1), \\
& M=\int_{-h / 2}^{h / 2} \sigma_{\theta}(z) z d z=D(1+\varepsilon)(a \kappa-1) / a .
\end{aligned}
$$

In order to formulate the problem in a non-dimensional base, two non-dimensional deformation variables $\xi$ (radial displacement, inward positive) and $\psi$ (circumferential rotation, counterclockwise positive) are introduced, i.e.

$$
\xi=1-\frac{r}{a}, \quad \psi=\phi-\theta
$$

Then, the membrane strain $\varepsilon$ and curvature $\kappa$ in Eqns. $(2.6,7)$ can be expressed as

$$
\begin{aligned}
& \varepsilon=\psi^{\prime}-\xi-\xi \psi^{\prime}+\frac{1}{2} \xi^{2}-\underline{\frac{1}{2} \xi^{2}\left(\psi^{\prime}-\xi\right)} \\
& a \kappa=1+\xi^{\prime \prime}+\xi-\left(\xi^{\prime} \psi^{\prime}\right)^{\prime}-\xi^{\prime \prime}\left(\psi^{\prime}-2 \xi\right)+\xi^{2}+\frac{1}{2} \xi^{2}
\end{aligned}
$$

It should be pointed out that the membrane strain given by Eqn.(2.14) is different from the one in the early publication (Eqn.11 in (Goodier and McIvor, 1964)). In the present membrane strain expression in Eqn.(2.14), additional third-order terms underlined in the expression are included in the higher order governing equations (up to third order) to avoid the numerical instability during the vibration mode transformation discussed in the introduction section. However, third-order terms are discarded completely in the curvature expression since they do not contribute to the same accuracy level in the final governing equations as their counterparts in membrane strain do.

After eliminating shear force $Q$ using Eqn.(2.5) and substituting accelerations using Eqn.(2.4a,b), with Eqns.(2.11-13) taken into account, the governing Eqns.(2.1,2) are rewritten using the two non-dimensional variables $\xi$ and $\psi$ as 


$$
\begin{aligned}
& \ddot{\xi}+(1-\underline{\xi}) \dot{\psi}^{2}+\left(1+\xi^{\prime \prime}\right)\left(\xi-\psi^{\prime}\right)-\psi^{\prime 2}+2 \psi^{\prime} \xi-\xi^{\prime} \psi^{\prime \prime}+\frac{1}{2} \xi^{\prime 2} \\
& +\frac{\psi^{\prime 2}\left(\xi+\xi^{\prime \prime}\right)+\frac{1}{2} \xi^{\prime 2}\left(\psi^{\prime}-3 \xi^{\prime \prime}\right)+\xi \xi^{\prime \prime}\left(\xi-\psi^{\prime}\right)+\psi^{\prime} \psi^{\prime \prime} \xi^{\prime}}{} \\
& +\alpha^{2}\left(\xi+2 \xi^{\prime \prime}+\xi^{(4)}+\underline{\Pi}\right)-\frac{a}{E_{1} h} p\left(1+\psi^{\prime}-\xi-\underline{\psi^{\prime} \xi}\right)=0, \\
& \ddot{\psi}-2(1+\underline{\xi}) \dot{\xi} \dot{\psi}+\xi^{\prime}-\psi^{\prime \prime}+\xi^{\prime}\left(2 \psi^{\prime}-\xi^{\prime \prime}\right)+\underline{\xi^{\prime} \psi^{\prime 2}+\frac{1}{2} \xi^{\prime 2} \psi^{\prime \prime}+\xi^{\prime} \xi^{\prime \prime}\left(2 \psi^{\prime}-3 \xi\right)} \\
& -\underline{\alpha^{2}\left[\left(\xi+\xi^{\prime \prime}\right)\left(\xi^{\prime}+\xi^{\prime \prime \prime}\right)+\xi^{\prime}\left(\xi+2 \xi^{\prime \prime}+\xi^{(4)}\right)\right]+\frac{a}{E_{1} h} p\left(1+\underline{\psi^{\prime}-\xi}\right) \xi^{\prime}=0,}
\end{aligned}
$$

where the dot above symbols indicates derivatives with respect to a new non-dimensional time variable $\tau=t \cdot c / a, \quad c$ is the propagation velocity of longitudinal waves and defined as $c=\sqrt{E_{1} / \rho}$. The aspect ratio $\alpha$ is

$$
\alpha=\frac{h}{2 \sqrt{3} a} .
$$

It should be pointed out that all the underlined terms in Eqns. $(2.16,17)$ are additional higher order terms compared with the governing equations in Goodier and McIvor's work (Goodier and McIvor, 1964, McIvor, 1966) and most of them are originated from the inclusion of the third order term in membrane strain given in Eqn.(2.14). The term $\Pi$ in Eqn.(2.16) is

$$
\begin{aligned}
\Pi= & -\left(\psi^{\prime} \xi^{\prime}\right)^{\prime}-\left(\psi^{\prime} \xi^{\prime}\right)^{\prime \prime \prime}+\psi^{\prime}\left(\xi-\xi^{(4)}\right)+\psi^{\prime \prime}\left(\xi^{\prime}-\xi^{\prime \prime \prime}\right)+\xi \psi^{\prime \prime \prime} \\
& +2 \xi\left(\xi^{\prime \prime}+\xi^{(4)}\right)+4 \xi^{\prime \prime \prime \prime}+2 \xi^{\prime \prime 2}+\frac{3}{2} \xi^{\prime 2}
\end{aligned}
$$

Given initial conditions, the governing equations $(2.16,17)$ can be solved numerically, which will be presented in section 4 .

\subsection{Explicit model for mode transformation}

Equations $(2.16,17)$ were employed in ( $\mathrm{Li}$ and Shi, 2008) to study the intermittent transformation between RBM and flexural vibration mode in single-walled carbon nanotubes where the numerical instability was first observed without further investigation. Experimental observations show that, when a nearly uniform impulse is applied on a thin cylindrical shell, it may undergo a motion of intermittent vibration mode transformations, i.e. the extensional mode and flexural 
mode occur intermittently through a nonlinear coupling (Zhu et al., 1997, Duffey and Romero, 2003, Li et al., 2008). Since the circular cylindrical shell is in a closed form, the two non-dimensional variables $\xi$ and $\psi$ must be periodic with respect to angle $\theta$ and accordingly can be expanded in following Fourier series

$\xi=a_{0}(\tau)+\sum_{n=1}^{\infty} a_{n}(\tau) \cos n \theta+\sum_{n=2}^{\infty} c_{n}(\tau) \cos n \theta$

$\psi=\quad \sum_{n=1}^{\infty} b_{n}(\tau) \sin n \theta+\sum_{n=2}^{\infty} d_{n}(\tau) \sin n \theta$

In above modal expansion of deformation variable $\xi$, only cosine terms are included since the mode transformations between pure extensional vibration mode (i.e. RBM) and the flexural modes represented by cosine terms are equivalent to the mode transformations between RBM and the flexural modes represented by sine terms. Accordingly only sine terms are included in the series expansion of $\psi$, which is due to the mathematical compatibility between the two expansions given in following Eqn.(2.22). A complete expansion with companion modes (sine terms) included can be found in (McIvor, 1966, Nayfeh and Raouf, 1987). In above expansions given by Eqns. $(2.20,21)$, the terms $a_{n}(\tau) \cos n \theta$ and $b_{n}(\tau) \sin n \theta$ represent extensional flexural deformation, whereas $c_{n}(\tau) \cos n \theta$ and $d_{n}(\tau) \sin n \theta$ represent inextensional flexural deformation. $c_{1}(\tau) \cos \theta$ and $d_{1}(\tau) \sin \theta$ are both discarded since they correspond to rigid translation. Consider pure extensional and pure flexural deformation of a middle surface element independently, as shown in Fig.2.4, one can get the extensionality and inextensionality conditions, respectively, as

$\xi^{\prime}=\psi \quad$ and $\quad \psi^{\prime}=\xi$

Consequently, the four unknown generalized deformation coordinate functions $a_{n}$ and $b_{n}, c_{n}$ and $d_{n}$ can be reduced to two by the following relations

$\left\{\begin{aligned} b_{i}=-i a_{i}, & i=1,2,3, \cdots \\ d_{j}=c_{j} / j, & j=2,3,4, \cdots\end{aligned}\right.$ 
ACCEPTED MANUSCRIPT

Next, Lagrange equations will be used to produce governing equations of the unknown coefficient functions $a_{0}(\tau), a_{n}(\tau)$ and $c_{n}(\tau)$, for which kinetic energy $T$ and potential energy $U$ need to be given first. For a cylindrical shell of unit longitudinal length, the kinetic energy $T$ and strain energy $U$, respectively, are given as

$T=\int_{0}^{2 \pi} \frac{1}{2} \rho a h\left[\left(\frac{\partial r}{\partial t}\right)^{2}+\left(r \frac{\partial \psi}{\partial t}\right)^{2}\right] d \theta=\frac{1}{2} E_{1} a h \int_{0}^{2 \pi}\left[\dot{\xi}^{2}+(1-\xi)^{2} \dot{\psi}^{2}\right] d \theta$

and

$U=\int_{0}^{2 \pi} \int_{-h / 2}^{h / 2} \frac{1}{2} \sigma_{\theta}(z) \varepsilon_{\theta}(z)(a+z) d \theta d z=\frac{1}{2} E_{1} a h \int_{0}^{2 \pi}\left[\varepsilon^{2}+\alpha^{2}(1+\varepsilon)^{2}(a \kappa-1)^{2}\right] d \theta$.

Substituting $\mathcal{E}$ and $\kappa$ given by Eqn.(2.14) and Eqn.(2.15) respectively into Eqn.(2.25), and then neglecting the fifth and higher order terms will yield

$U=\frac{1}{2} E_{1} a h\left\{\int_{0}^{2 \pi}\left[\left(\psi^{\prime}-\xi\right)^{2}+\left(\psi^{\prime}-\xi\right)\left(\xi^{2}-2 \xi \psi^{\prime}\right)+\alpha^{2}\left(\xi+\xi^{\prime \prime}\right)^{2}\right] d \theta+\Delta\right\}$

where

$\Delta=\int_{0}^{2 \pi}\left[2 \alpha^{2}\left(\psi^{\prime}-\xi\right)\left(\xi+\xi^{\prime \prime}\right)^{2}+\frac{1}{4}\left(\xi^{\prime 2}-2 \xi \psi^{\prime}\right)^{2}-\xi^{\prime 2}\left(\psi^{\prime}-\xi\right)^{2}\right] d \theta$

In the expression of strain energy in Eqn.(2.26), in which, since $\alpha^{2}<<1$, the term $\int_{0}^{2 \pi} \alpha^{2}\left(\xi+\xi^{\prime \prime}\right)^{2} d \theta$ can be treated as a third-order small term. The term $\Delta$ represents contributions to the degree of fourth order and is on at least one order smaller when compared to the second- and third-order terms for small deformation. As a result, the contributions from this term were discarded completely or partially in earlier publications (Goodier and McIvor, 1964, McIvor, 1966, Lindberg, 1974). However, it will be shown in next section that it is essential to include a complete fourth-order term in strain energy to guarantee the numerical stability of the solution.

The Lagrange equation for any generalized coordinate $q_{i}$ of a general mechanical system in absence of external loadings is 
$\frac{\partial}{\partial \tau}\left(\frac{\partial T}{\partial \dot{q}_{i}}\right)-\frac{\partial T}{\partial q_{i}}+\frac{\partial U}{\partial q_{i}}=0, \quad i=1,2,3, \cdots$

Substituting series of the two non-dimensional variables $\xi$ and $\psi$ given by Eqns. $(2.20,21)$ into energy integrals given by Eqns.(2.24, 26) respectively, then inserting the results of kinetic energy $T$ and strain energy $U$ into Lagrange Eqns.(2.28) while taking $a_{0}(\tau), a_{n}(\tau)$ and $c_{n}(\tau)$ as the generalized coordinate $q_{i}$ in sequence, will give the final governing differential equations of $a_{0}(\tau), a_{n}(\tau)$ and $c_{n}(\tau)$, i.e.

$$
\begin{aligned}
& \ddot{a}_{0}+a_{0}-\frac{1}{4} \sum_{n}^{\infty}\left(n^{2}-2\right) c_{n}^{2}+\frac{1}{2} \frac{\partial \Delta}{\partial a_{0}}=0 \\
& \ddot{a}_{n}+\left(n^{2}+1\right) a_{n}+\alpha^{2} \frac{\left(n^{2}-1\right)^{2}}{n^{2}+1} c_{n}-\frac{1}{n^{2}+1} a_{0}\left[n^{2}\left(2 n^{2}+5\right) a_{n}+\left(n^{2}-2\right) c_{n}\right] \\
& +\frac{1}{4} \sum_{m=1}^{\infty}\left\{[2-m(m+n)] c_{m} c_{m+n}+[2-m(m-n)] c_{m} c_{|m-n|}\right\}+\frac{1}{n^{2}+1} \frac{\partial \Delta}{\partial a_{n}}=0, \\
& \ddot{c}_{n}+\alpha^{2} \frac{n^{2}\left(n^{2}-1\right)^{2}}{n^{2}+1} c_{n}-\frac{n^{2}\left(n^{2}-2\right)}{n^{2}+1} a_{0} c_{n}+\frac{n^{2}}{2\left(n^{2}+1\right)} \sum_{m=1}^{\infty}\left\{[2-n(m+n)] c_{m+n}\right. \\
& \left.\quad+[2+n(m-n)] c_{|m-n|}\right\}\left(m^{2}+1\right) a_{m}+\frac{n^{2}}{n^{2}+1} \frac{\partial \Delta}{\partial c_{n}}=0 .
\end{aligned}
$$

In Eqns.(2.29-31), it should be noted that (1) The inextensional flexural deformation $\left(c_{n}\right)$ is assumed to be much stronger than the extensional flexural deformation $\left(a_{n}\right)$, as a result that the extensional flexural terms have been discarded completely in Eqns.(2.29-31) where inextensional terms also appear in the summation. (2) The coefficient of the linear term $a_{0}$ is simply unity in its governing equation (i.e. Eqn.(2.29)). This indicates that the circular frequency of $a_{0}$ will be unity, which is directly resulted from the choice of non-dimensional time variable $\tau=t \cdot c / a$. (3) It can be seen that the linear part of Eqns.(2.29-31) is not diagonal, which does not imply that the series expansions of $\xi$ and $\psi$ in Eqns.(2.20, 21) are not normal mode expansions. It is simply due to that the flexural deformation corresponding to cosine terms is decomposed into extensional and inextensional parts, i.e. $a_{n} \cos \theta$ and $c_{n} \cos \theta$. (4) It is difficult to explicitly 
ACCEPTED MANUSCRIPT

implement the integration in term $\Delta$ when strain energy $U$ is substituted into the Lagrange equation, therefore $\Delta$ is kept in above governing equations representing the contributions from the fourth-order terms. As a result, it will recur to numerical method to carry out the integration in the term $\Delta$ when numerical solutions of $a_{0}(\tau), a_{n}(\tau)$ and $c_{n}(\tau)$ are sought for governing Eqns.(2.29-31).

About the initial conditions for Eqns.(2.29-31), since we focus on mode transformations initiated from a nearly uniform RBM mode, the same initial conditions of $\xi$ and $\psi$ used in (McIvor, 1966) are introduced for convenience of comparison. Thus, the initial conditions are

$$
\begin{aligned}
& \dot{\xi}(\theta, 0)=\frac{1}{c}\left(v_{0}+\sum_{n=1}^{\infty} v_{n} \cos n \theta\right) \\
& \dot{\psi}(\theta, 0)=-\frac{v_{1}}{c} \sin \theta, \quad \xi(\theta, 0)=\psi(\theta, 0)=0 .
\end{aligned}
$$

In above initial conditions $v_{0}$ stands for a perfectly uniform radial velocity of the wall of the cylinder and $v_{n}(n=1,2,3, \cdots)$ represent imperfections in this uniformity which are normally at least two orders smaller than $v_{0}$. Correspondingly, the initial conditions for the governing Eqns.(2.29-31) of $a_{0}(\tau), a_{n}(\tau)$ and $c_{n}(\tau)$ can be determined as

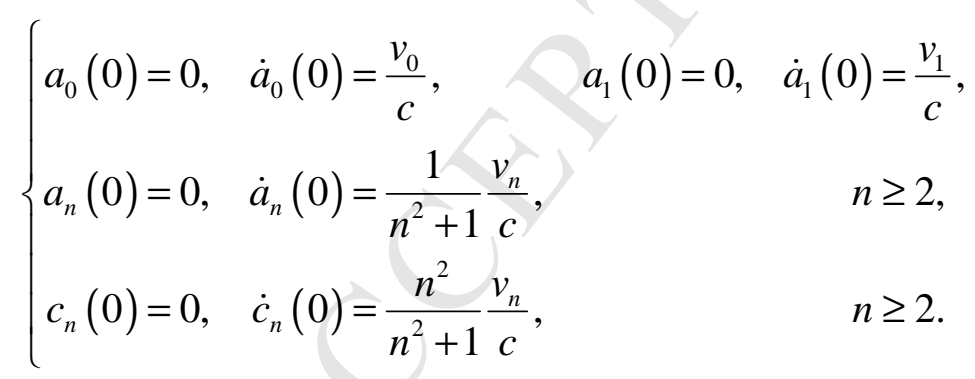

The fourth order Runge-Kutta method (RK4) is employed to solve the differential Eqns.(2.29-31) under initial conditions given in Eqn.(2.34).

Now two sets of governing equations have been obtained for the cylindrical shell under radial impulse: one is named the implicit model governed by Eqns.(2.16,17) and the other is called explicit model governed by Eqns.(2.29-31). In general, the implicit model and explicit model for mode transformation in cylindrical shells are not strictly equivalent. In fact, it is difficult to give a 


\section{ACCEPTED MANUSCRIPT}

precise equivalence between the two models because they are established following different approaches. But, regarding their accuracy, the explicit model may be one order higher in accuracy than the implicit model. Though the same basic strain expressions given by Eqns. $(2.8,14)$ are used in both models, truncated terms up to order four are kept in the strain energy in Eqn.(2.26), and therefore, in the final governing Eqns.(2.29-31) of the explicit model. However, truncated terms up to order only three are kept in the final governing Eqns $(2.16,17)$ of implicit model. But, it should be pointed out that the accuracy of the explicit model is sacrificed because only a limited number of terms can be included in the truncated series expansions in Eqns. $(2.20,21)$ when numerical solutions are sought. Furthermore, it is also adverse to the accuracy of explicit model where the extensional flexural terms $\left(a_{n}\right)$ in the final governing equations are discarded when they appear in summation together with the corresponding inextensional term $\left(c_{n}\right)$.

The numerical solutions of the explicit model will be given in section 3 with focus on the excitation of inextensional flexural modes and vibration stability while the numerical solutions of the implicit model will be given in section 4 with focus on stress growth.

\section{Numerical results of different explicit models}

In section 2.2 an explicit model for mode transformation in cylindrical shell is established. When different imperfections are introduced in the initial conditions (correspondingly different flexural terms will need to be included in the model) or terms of different orders are included in the model, the explicit model given by governing Eqns.(2.29-31) combined with initial conditions in Eqns.(2.32-34) will be defined for some specific cases. In this section explicit model in different cases will be introduced and discussed.

When the initial imperfections to uniform impulse are discarded completely, the cylindrical shell is assumed to be subjected to perfectly uniform radial impulse. Correspondingly, $v_{n}=0, n \geq 1$ in Eqns.(2.32-34), then $a_{n} \equiv 0$ and $c_{n} \equiv 0$, and a simple analytical solution of Eqn.(2.29) can be obtained

$a_{0}=v_{0} c^{-1} \sin \tau$. 
This solution indicates a vibration of simple in-and-out radial "breathing" mode (RBM) or pure extensional mode. Accordingly, cross-section of the cylindrical shell will remain circular at any instant. However, when imperfections are taken into account in the initial conditions, though their magnitudes are at least two orders smaller than that of the fundamental RBM vibration, numerical simulations in following sections will show that, as time increases, one or more inextensional flexural modes will be excited to an amplitude of the same order of RBM and the cylindrical shell will vibrate in coupled extensional and flexural modes. In following examples, in addition to the focus on the excitation of inextensional flexural modes, stability of the vibration, especially the effect of the fourth-order term $\Delta$ on the stability of numerical solutions, will also be studied.

\subsection{Goodier and McIvor's model}

When the fourth-order term $\Delta$ and the third-order term with coefficient $\alpha^{2}$ are both omitted completely in the strain energy integral in Eqn.(2.26), and only inextensional flexural vibration is considered, i.e., $a_{n} \equiv 0, n=1,2,3, \cdots$ in Eqns.(2.20, 21), then the governing Eqns.(2.29-31) will be reduced to

$$
\begin{aligned}
& \ddot{a}_{0}+a_{0}-\frac{1}{4} \sum_{n}^{\infty}\left(n^{2}-2\right) c_{n}^{2}=0, \\
& \ddot{c}_{n}+\alpha^{2} \frac{n^{2}\left(n^{2}-1\right)^{2}}{n^{2}+1} c_{n}-\frac{n^{2}\left(n^{2}-2\right)}{n^{2}+1} a_{0} c_{n}=0
\end{aligned}
$$

which are respectively the same as Eqn.(43) and (44) in (Goodier and McIvor, 1964). For this group of governing Eqns.(3.2,3), initial conditions of $\xi$ and $\psi$ in Eqns.(2.32, 33) should be modified as [Note: this is due to the necessity of mathematical compatibility. Detailed discussions can be referred to the reference (Goodier and McIvor, 1964)]

$$
\left\{\begin{array}{l}
\xi(\theta, 0)=0, \quad \dot{\xi}(\theta, 0)=\frac{v_{0}}{c}\left(1+\sum_{n=2}^{\infty} \gamma_{n} \cos n \theta\right), \\
\psi(\theta, 0)=0, \quad \dot{\psi}(\theta, 0)=\frac{v_{0}}{c} \sum_{n=2}^{\infty} \frac{\gamma_{n}}{n} \sin n \theta, \quad\left|\gamma_{n}\right| \leq 0.01, \quad n=1,2,3, \cdots
\end{array}\right.
$$

Accordingly, initial conditions for $a_{0}$ and $c_{n}$ can be determined as 
$\left\{\begin{array}{l}a(0)=0, \quad \dot{a}(0)=\frac{v_{0}}{c}, \\ c_{n}(0)=0, \quad \dot{c}_{n}(0)=\frac{v_{0}}{c} \gamma_{n}, \quad n=1,2,3, \cdots .\end{array}\right.$

For a cylindrical shell with fixed aspect ratio $a / h$, numerical results show that when the initial radial velocity $v_{0}$ reaches a sufficiently large value, one or more flexural vibration modes $c_{n}$ will be excited to amplitude of the same or even higher order of RBM $a_{0}$. This phenomenon can be explained by the Mathieu equation and Mathieu stability diagram (Mclachilan, 1947, Struble, 1962, Goodier and McIvor, 1964).

A numerical example is shown in Fig.3.1, in which $a / h=20.4$ and initial RBM velocity $v_{0} / c=0.005$. In this and following examples, for the purpose of comparison, the same aspect ratio $a / h=20.4$ is chosen as in the numerical example shown in Fig.6 in (Goodier and McIvor, 1964). It might be necessary to point out that, in all numerical examples thereafter, the imperfections [i.e. $\gamma_{n}$ in Eqn.(3.4) or $v_{n}$ in Eqns.(2.32-34), $n=1,2,3, \cdots, N$, where $N$ is the maximum mode number of flexural modes $a_{n}$ and $c_{n}$ included in series given by Eqn. $(2.20,21)$ when numerical solutions are sought] are produced stochastically with the maximum amplitude fixed at exactly two orders smaller than the initial RBM vibration velocity (i.e. $\left\|v_{n}\right\| \leq v_{0} / 100$ ). For the cylindrical shell under the initial condition $\left(v_{0} / c=0.005\right)$, it is shown in Fig.3.1 that inextensional flexural mode $c_{6}$ is excited. Clearly, the cylindrical shell experienced a nonlinearly-coupled vibration of the extensional and flexural modes and the intermittent transformation of vibration modes and energy transfer happen (Goodier and McIvor, 1964). But, for the same cylindrical shell, when the initial radial impulse is stronger than some critical amplitude or equivalently when the initial velocity $v_{0}$ reaches a critical value, as discussed in (Lindberg, 1974), numerical solution can easily begin divergent after a period of time, or the vibration can become instable soon. In (Lindberg, 1974), this critical value of initial velocity $v_{0}$ was determined by the condition that stability parameter $p=v_{0} /(\alpha c)=1 / 2$. Then for the cylindrical shell shown in Fig.3.1, the critical initial velocity can 
be determined as $v_{0}=0.00721 c$. When the initial velocity reaches $0.0075 c$, the numerical results of RBM $a_{0}$ and inextensional flexural mode $c_{6}$ are shown in Fig.3.2. It is evident that, with the increase of time, the periodic mode transformations between $a_{0}$ and $c_{6}$ start to fail and then the vibration becomes instable. Since this vibration instability still occurs at approximately the same moment (with slightly different vibration amplitude) when time step is refined from 0.1 to 0.01 (in non-dimensional time $\tau$ domain), it is reasonable to say that this vibration instability is not caused by numerical errors in the RK4 method, but due to the intrinsic physical instability in the formulation of the problem. However, if the fourth-order term $\Delta$ in strain energy integral in Eqn.(2.26) is included together with the assumption that only one flexural vibration mode $c_{M}$ is possibly excited (actually from last example we know that $M=6$, and thus, only this specific flexural mode is included in the series expansions given in Eqn.(2.20, 21)), then the governing Eqns.(3.2,3) will be upgraded to

$$
\begin{aligned}
& \ddot{a}_{0}+a_{0}- \frac{1}{4}\left(M^{2}-2\right) c_{M}^{2}+\frac{1}{2} a_{0} c_{M}^{2}=0, \\
& \ddot{c}_{M}+\alpha^{2} \frac{M^{2}\left(M^{2}-1\right)^{2}}{M^{2}+1} c_{M}-\frac{M^{2}\left(M^{2}-2\right)}{M^{2}+1} a_{0} c_{M} \\
&+\frac{M^{2}}{M^{2}+1}\left[\frac{3}{8}\left(M^{4}-\frac{4}{3} M^{2}+4\right) c_{M}^{3}+a_{0}^{2} c_{M}\right]=0 .
\end{aligned}
$$

For the same problem shown in Fig.3.2, numerical results based on Eqns.(3.6, 7) are shown in Fig.3.3, which clearly shows that the cylindrical shell has undergone a stable motion for a much longer period with periodic vibration mode transformations. Therefore, it can be seen that the fourth-order term $\Delta$ is crucial in the governing equations to guarantee a stable vibration solution.

\subsection{McIvor's model}

An explicit model with more generalized governing equations in (McIvor, 1966) than those in (Goodier and McIvor, 1964) was established to explore the growth of membrane and flexural stresses for a thin cylindrical shell under nearly uniform radial impulse. Similarly, the fourth-order term $\Delta$ and the third-order term with coefficient $\alpha^{2}$ were both discarded completely in the strain energy integral in Eqn.(2.26). The governing equations in the model 


\section{ACCEPTED MANUSCRIPT}

(McIvor, 1966) is a special case of Eqns.(2.29-31). In the present study, in addition to the fourth-order term $\Delta$, the term $a_{0} a_{n}$ in Eqn.(2.30) was also dropped since extensional flexural terms $a_{n}$ are treated as at least one order smaller than inextensional flexural terms $c_{n}$. With this group of governing equations, more general initial imperfections as described by Eqns.(2.32-34) can be investigated than the earlier model (Goodier and McIvor, 1964).

For this set of governing equations, Fig.3.4 shows the numerical results of the same cylindrical shell under the same initial velocity $v_{0} / c=0.005$ as in Fig.3.1. From Fig.3.4, it can be seen that the cylindrical shell experiences a periodic transformations between the extensional and flexural modes in the early stage. However, as time $\tau$ passes 300 , the shell begins to vibrate irregularly and becomes divergent very soon. Therefore, the vibration instability occurs again at a much shorter time compared with the stable period in Fig.3.1 according to Goodier and McIvor's model. It implies that the inclusion of extensional flexural mode imperfections has strong negative effect on the vibration stability.

\subsection{Present explicit model}

Though McIvor's model corresponds to a more general circumstance of initial impulse imperfections, numerical results show that when the fourth-order term is not included in the strain energy, the vibration can become instable soon after the impulse is imposed. However, it has been shown in Fig.3.3 that stable vibration or steady intermittent mode transformations can be achieved when the fourth-order term is included in the governing Eqns.(3.6, 7), in which only the inextensional flexural imperfection is considered and only one inextensional flexural mode is assumed. In the present explicit model, numerical solutions for vibration and mode transformations will be sought based on the governing Eqns.(2.29-31) under the general initial conditions given in Eqn.(2.34). It should be noted that the fourth-order term $\Delta$, which is included in Eqns.(2.29-31), is calculated according to Eqn.(2.27) with standard Gaussian integration method.

The same cylindrical shell under the exactly same initial conditions (which means that the initial impulse imperfections are exactly the same) as those used in Fig.3.1 and Fig.3.4 is investigated with present explicit model and the results are shown in Fig.3.5. It can be seen that, compared 
with the vibration history based on McIvor's model as shown in Fig.3.4, stable vibration lasts more than twice the stable period predicted by McIvor's model and steady intermittent vibration transformations between RBM $a_{0}$ and the inextensional flexural mode $c_{6}$ can be observed.

To further investigate the causes and nature of the numerical instability when the fourth-order term is omitted in the strain energy, numerical results of the two lowest vibration modes $c_{2}$ and $c_{3}$ and energy history for the formulations with and without the fourth-order term included in strain energy are presented in Figs.3.6 and 3.7, respectively. The dashed curves in Fig.3.6 correspond to the case when the fourth-order term $\Delta$ is not included in strain energy. The amplitudes of the two lowest modes $c_{2}$ and $c_{3}$, which are supposed to be much smaller in amplitude than both the principle $\mathrm{RBM} a_{0}$ and the major excited flexural mode $c_{6}$, increase sharply to a level unbounded after certain period. This leads to an abrupt deviation of the RBM $\left(a_{0}\right)$ and the excited inextensional flexural mode $\left(c_{6}\right)$ from their periodic mode transformations. When the fourth-order term $\Delta$ is included in the strain energy integral in Eqn.(2.26), the predicted responses of $c_{2}$ and $c_{3}$ (solid curves in Fig.3.6) are as expected to be periodic vibration with the amplitude at least one order smaller than that of $a_{0}$ and $c_{6}$. This suppression of amplitude increase on low flexural modes can be explained by the energy histories with and without the fourth-order term included in the strain energy as shown in Fig.3.7. Since there is no energy fluxed in or out of the whole system, the total energy of the system (kinetic energy $T$ given by Eqn.(2.24) and strain energy $U$ given by Eqn.(2.26)) should remain constant as shown by the solid curve in Fig.3.7, which is predicted when the fourth-order term $\Delta$ is included in the strain energy in Eqn.(2.26). However, the dashed curve in Fig.3.7 shows that, when the fourth-order term $\Delta$ is not included in the strain energy, the total energy $E$ remains constant for a period of time before it departs dramatically from the initial value $E_{0}$. By checking the strain energy expression in Eqn.(2.26), it is found that the fourth-order term is a key factor to guarantee the strain energy to be positively definite when the third-order term becomes negative and reaches the same order of the second-order terms after the inextensional flexural modes have been excited a few times during vibration. 


\section{ACCEPTED MANUSCRIPT}

The response at a typical point $(a, \theta=0)$ is investigated with the fourth-order term included in strain energy in Eqn.(2.26) and the results in frequency domain are shown in Fig.3.8. Two distinct frequency peaks can be seen in graph (c), which correspond to the RBM $a_{0}$ and the excited major flexural mode $c_{6}$ as plotted individually in insets in the graphs (a) and (b). It is shown that the frequency of the excited flexural mode is nearly half the RBM frequency, which can be explained by the 1:2 internal resonance between the major excited flexural mode and the principle RBM in thin-walled structures in the case of either free vibrations (Goodier and McIvor, 1964, Touze and Thomas, 2006) or forced vibrations (Thomas et al., 2005, Thomas et al., 2007, Amabili, 2008, Camier et al., 2009).

\section{Numerical results of implicit model}

For the original differential Eqns. $(2.16,17)$ of $\xi$ and $\psi$, numerical solutions can be obtained by direct integration in the time $(\tau)$ domain with the fourth-order Runge-Kutta method and meanwhile using finite difference method in the space $(\theta)$ domain (use standard fourth-order central differences, (Rappaz et al., 2003)). In order to compare implicit model with the explicit model presented in last section, membrane stress $\sigma_{m}$ and flexural stress $\sigma_{f}$ at point $(a, \theta=0)$ are calculated with numerical solutions of $\xi$ and $\psi$. With the definitions of normal force $S$ and moment $M$ given by Eqns.(2.11,12), membrane stress $\sigma_{m}$ is defined as the average stress on the side where normal force $S$ is applied (see Fig.2.2), and flexural stress $\sigma_{f}$ is defined as the maximum stress due to the moment $M$ on the same side, i.e.

$$
\left\{\begin{array}{l}
\sigma_{m}=\frac{S}{h} \approx E_{1}\left[\xi-\psi^{\prime}+\alpha^{2}\left(\xi+\xi^{\prime \prime}\right)\right], \\
\sigma_{f}=\frac{M}{D} \cdot \frac{h}{2} \approx E_{1}\left[\sqrt{3} \alpha\left(\xi+\xi^{\prime \prime}\right)\right] .
\end{array}\right.
$$

In order to compare predictions based on different models, exactly the same initial conditions are used equivalently for $\xi$ and $\psi$, and for $a_{0}, a_{n}$ and $c_{n}$ through Eqns.(2.32-34). All the following numerical results are obtained for the same cylindrical shell with aspect ratio 
$a / h=20.4$ but with varying initial RBM velocity $\left(v_{0}\right)$ in Eqns.(2.32-34). The initial velocity imperfections $v_{n}$ in Eqns.(2.32-34) are produced using the same way in previous section and fixed at two orders smaller than $v_{0}$. Before we compare the numerical results from the two models, the second-order (without those underlined terms) and third-order (with those underlined terms) governing Eqns.(2.16,17) are used to predict the stresses and energy histories for the cylindrical shell under $v_{0} / c=0.005$. It can be seen from Fig.4.1 that, the second- and the third-order governing equations predict quite different stress evolutions after the moment when the flexural stress reaches its maximum at time around $\tau=100$ [i.e. around the first group of peaks in Fig.4.1 (b) and (d)]. When compared to the uniform periodic transformation of membrane and flexural stresses during the total time span (600) shown in (c) and (d), the transformations of stresses shown in (a) and (b) occur at a higher frequency and are not uniform at all during the period, especially in the last transformations. This difference can still be observed even when the time step is much smaller. It seems that the period of mode or stress transformation is stretched considerably longer in the third-order formulation of the problem. As energy is concerned, the total energy $(E)$ values based on the two formulations are shown in Fig.4.2. For an insulated system, the total energy should be constant. But the curves (i) and (ii) in Fig.4.2 clearly show that energy deviates from its initial value $E_{0}$ around flexural stress peaks. It can be seen that the third-order formulation produces a much less energy deviation than the second-order one. This, together with the uniform pattern of stress transformations shown in Fig.4.1(c) and (d), evidently indicates that the third-order formulation of implicit model provides satisfactorily higher precision than the second-order one. The energy curve in Fig.4.2(ii) shows that the relative error of the numerical results from the third-order formulation is within $2 \%$, hence thereafter all the numerical results based on the explicit model will use the third-order governing equations. Figure 4.3 shows the frequency spectra of stresses at point $(a, \theta=0)$ predicted by the third-order implicit model. Membrane stress, which is shown as inset in the spectrum (a) in Fig.4.3, should have the frequency of RBM since $\psi^{\prime}-\xi$ in Eqn.(4.1) is the dominant term in membrane stress and it corresponds to extensional vibration mode. Spectrum (b) in Fig.4.3 indicates that the flexural stress vibrates at half the frequency of membrane stress, 
which agrees with the frequency result given by the explicit model shown in Fig.3.8 since the flexural stress essentially comes from the flexural vibration.

The flexural stress for the same cylindrical shell under $v_{0} / c=0.005$ is calculated to show the differences between the implicit model (third-order) and the present explicit model. Since the numerical solution based on the McIvor's model will become divergent after time $\tau$ slightly larger than 300, this model is not included in the comparison. The two graphs Fig.4.4(a) and (b) are the flexural stress responses from the implicit and explicit models, respectively. It can be seen that the flexural stresses produced by the two models follow very similar evolution patterns, especially that the first group of stress peaks (around time $\tau=100$ ) given by the two models shown in Fig.4.4(c) match very well. However, after the first group of peaks shown in Fig.4.4(c), the flexural stress based on the explicit model [dashed line shown in Fig.4.4(d)] reaches the subsequent peaks earlier than those based on the implicit model [solid line in the same graph]. It seems that, in the second group of peaks, the phase of the flexural stress predicted by the explicit model is shifted to the left in comparison with the phase of the flexural stress predicted by the implicit model. Since each pair of stress peak amplitudes among the first [Fig.4.4(c)] and second groups [Fig.4.4(d)] predicted by the two models match well, and within each group of peaks, the frequency or period of stress vibrations is very close to each other, the phase-shifting phenomenon occurring in the second and subsequent groups of stress peaks can not be explained by the amplitude-frequency characteristics (AFCs) for free and forced vibrations of thin-walled structures (Kubenko and Koval'chuk, 1998). It is conjectured that the truncated series expansion of the deformation variable in explicit model limited the flexural modes to be coupled with the principle RBM, which leads to stronger coupling between the dominantly excited mode $c_{M}$ and the principle RBM $a_{0}$. Hence, the energy loss from RBM $a_{0}$ will be fully transferred to $c_{M}$ as shown in Fig.3.3. Therefore, the major excited flexural mode can get larger share of energy transferred from the RBM and reaches its peaks earlier. In early stage, the major excited flexural term is the dominant term and other flexural terms are so small that they can be neglected; the major excited flexural mode is almost identical to the deformation variable itself, which explains why the flexural stresses from two different models are almost identical in Fig.4.4(c). However, with the increase of time, other minor flexural modes are gradually excited and their amplitudes 


\section{ACCEPTED MANUSCRIPT}

are gradually increased. Their magnitudes, although still relatively low, become larger than their magnitudes in the early stage. Therefore, these minor flexural modes take more energy from the major excited flexural mode and cause the phase shift observed in Fig.4.4(d). It is expected that this phase shift will become larger in further later group of stress peaks.

It should be noted that the inclusion of the fourth-order term in strain energy cannot guarantee the elimination of the numerical divergence in this vibration mode transformation problem. The reason is simply that, if the initial velocity is rather large, one or more flexural vibration modes (including lower flexural modes in general cases) may be excited, which may reach amplitudes higher than the RBM amplitude, resulting in non-positive definite strain energy. Theoretically, even higher-order terms may be required in the expression of strain energy in order to keep the strain energy positively-definite throughout the whole vibration or mode transformation process. However, practically, the initial velocity is limited by the elastic limit of the shell material. According to Eqn.(2.14), when RBM is considered in the early vibration phase of a thin-walled cylindrical shell, the circumferential strain is expressed by

$$
\varepsilon_{\theta}=\frac{w}{a}
$$

where $w$ is the radial displacement. Based on energy conservation, the relationship between the initial velocity and the maximum circumferential strain is

$$
\frac{v_{0}}{c}=\frac{w_{\max }}{a}=\varepsilon_{\theta \max } .
$$

Thus, the non-dimensional number $\frac{v_{0}}{c}$ is limited by the yield strain of the material, i.e. $\varepsilon_{\theta \max } \leq \varepsilon_{y}=\frac{\sigma_{y}}{E}$, where $\sigma_{y}$ is the yield stress of the shell material. This value is normally less that $1 \%$ for engineering materials. Thus, we expect that the inclusion of fourth-order term in the present explicit model is sufficient for avoiding numerical divergence in most practical problems.

\section{Conclusions}

An implicit model and an explicit model in this analytical study are established with focus on the stability of vibration mode transformations between extensional and flexural modes in 


\section{ACCEPTED MANUSCRIPT}

thin-walled cylindrical shells. Vibration mode transformation occurs when the initial radial velocity (or the impulse) is large enough. However, when the fourth-order term in strain energy is discarded, numerical solutions based on explicit model may become divergent, which is found to be associated with the non-positive definite strain energy when the negative third-order terms are accumulated to an amplitude close to the order of the second-order terms in strain energy. It is demonstrated that the inclusion of fourth-order terms in the strain energy function can practically eliminate the numerical instability in the vibration mode transformation in thin-walled cylindrical shells.

The same 2:1 internal resonance is revealed for the mode transformation based on both implicit and explicit models. As flexural stress growth is concerned, the stress patterns predicted by both models match reasonably well. Two models predicted very close flexural stresses in the first group of peaks. However, in later group of stress peaks, phase shift gradually appears between the predicted flexural stresses based on implicit and explicit models. This can be explained by the limited number of terms included in the series expansions of explicit model. When only a limited number of terms are taken into account in the explicit model, compared to the inclusion of infinite number of terms in implicit model, the excited dominant flexural mode in the explicit model has a larger share of energy, and therefore, can be excited easily, especially in the later stage of vibration.

\section{Acknowledgements}

The author M.X. SHI acknowledges the financial support from Dorothy Hodgkin Postgraduate Awards (EPSRC-Shell Award).

\section{References}

[1] Amabili, M. (2003) A Comparison of Shell Theories for Large-Amplitude Vibrations of Circular Cylindrical Shells: Lagrangian Approach. Journal of Sound and Vibration, 264, 1091-1125.

[2] Amabili, M. (2008) Nonlinear Vibrations and Stability of Shells and Plates, Cambridge, Cambridge University Press.

[3] Amabili, M. and Paidoussis, M.P. (2003) Review of Studies on Geometrically Nonlinear Vibrations and Dynamics of Circular Cylindrical Shells and Panels, with and without Fluid-Structure Interaction. Applied Mechanics Reviews, 56, 349-381. 
[4] Amabili, M., Pellicano, F. and Paidoussis, M.P. (1999) Further Comments on Nonlinear Vibrations of Shells. Journal of Fluids and Structures, 13, 159-160.

[5] Amabili, M., Pellicano, F. and Vakakis, A.F. (2000) Nonlinear Vibrations and Multiple Resonances of Fluid-Filled, Circular Shells, Part 1: Equations of Motion and Numerical Results. Journal of Vibration and Acoustics-Transactions of the Asme, 122, 346-354.

[6] Byrne, R. (1944) Theory of Small Deformation of a Thin Elastic Shell. Seminar reports in Mathematics, University of California～Publications in Mathematics, N.S. 2,. 103-152.

[7] Camier, C., Touzé, C. and Thomas, O. (2009) Non-Linear Vibrations of Imperfect Free-Edge Circular Plates and Shells. European Journal of Mechanics - A/Solids, 28, 500-515.

[8] Donnell, L.H. (1938) A Discussion of Thin Shell Theory. Proceedings of the Fifth International Congress on Applied Mechanics.

[9] Dowell, E.H. (1967) On Nonlinear Flexural Vibrations of Rings. Aiaa Journal, 5, 1508-\&.

[10] Dowell, E.H. (1998) Comments on the Nonlinear Vibrations of Cylindrical Shells. Journal of Fluids and Structures, 12, 1087-1089.

[11] Dowell, E.H. and Ventres, C.S. (1968) Modal Equations for the Nonlinear Flexural Vibrations of a Cylindrical Shell. International Journal of Solids and Structures, 4, 975-991.

[12] Duffey, T.A. and Romero, C. (2003) Strain Growth in Spherical Explosive Chambers Subjected to Internal Blast Loading. International Journal of Impact Engineering, 28, 967-983.

[13] Evensen, D.A. (1963) Some Observations on the Nonlinear Vibration of Thin Cylindrical Shells. Aiaa Journal, 1, 2857-2858.

[14] Evensen, D.A. (1964) Non-Linear Flexural Vibrations of Thin-Walled Circular Rings. Phd Thesis. Pasadena, CA, California Institute of Technology.

[15] Evensen, D.A. (1966) Nonlinear Flexural Vibrations of Thin Circular Rings. Journal of Applied Mechanics, 33, 553-560.

[16] Evensen, D.A. (1967) Nonlinear Flexural Vibrations of Thin-Walled Circular Cylinders. Nasa Tn D-4090.

[17] Evensen, D.A. (1974) Non-Linear Vibrations of Circular Cylindrical Shells. IN FUNG, Y. C. andSECHLER, E. E. (Eds.)) Thin Walled Structures: Theory, Experiment and Design. Englewood Cliffs, NJ: Prentice-Hall.

[18] Evensen, D.A. (1999) Nonlinear Vibrations of Cylindrical Shells - Logical Rationale. Journal of Fluids and Structures, 13, 161-164. 
[19] Flugge, W. (1934) Statik Und Dynamic Der Schalen, Berlin, Julius Springer.

[20] Goodier, J.N. and Mcivor, I.K. (1964) The Elastic Cylindricall Shell under Nearly Uniform Radial Impulse. Journal of Applied Mechanics, 31, 259-266.

[21] Grigolyuk, E.I. (1955a) Nonlinear Vibrations and Stability of Shallow Shells and Rods. Izv. Akad. Nauk SSSR Mekh. Mashinostr., 3, 33-68.

[22] Grigolyuk, E.I. (1955b) Vibrations of Circular Cylindrical Panels Subjected to Finite Deflections. Prikl. Mat. Mekh, 19, 376-382.

[23] Krysko, V.A., Awrejcewicz, J. and Saveleva, N.E. (2008) Stability, Bifurcation and Chaos of Closed Flexible Cylindrical Shells. International Journal of Mechanical Sciences, 50, 247-274.

[24] Kubenko, V.D. and Koval'chuk, P.S. (1998) Nonlinear Problems of the Vibration of Thin Shells (Review). International Applied Mechanics, 34, 703-728.

[25] Leissa, A.W. (1984) Non-Linear Analysis of Plates and Shell Vibrations. Proceedings of the Second International Conference on Recent Advances in Structure Dynamics.

[26] Li, Q.M., Dong, Q. and Zheng, J.Y. (2008) Strain Growth of the in-Plane Response in an Elastic Cylindrical Shell. International Journal of Impact Engineering, 35, 1130-1153.

[27] Li, Q.M. and Shi, M.X. (2008) Intermittent Transformation between Radial Breathing and Flexural Vibration Modes in a Single-Walled Carbon Nanotube. Proceedings of the Royal Society a-Mathematical Physical and Engineering Sciences, 464, 1941-1953.

[28] Liew, K.M., Lim, C.W. and Kitipornchai, S. (1997) Vibration of Shallow Shells: A Review with Bibliography. Applied Mechanics Reviews, 50, 431-444.

[29] Lindberg, H.E. (1974) Stress Amplification in a Ring Caused by Dynamic Instability. Journal of Applied Mechanics-Transactions of the Asme, 41, 392-400.

[30] Lovell, E.G. and Mcivor, I.K. (1969) Nonlinear Response of a Cylindrical Shell to an Impulsive Pressure. Journal of Applied Mechanics, 36, 277-284.

[31] Lur'ye, A.I. (1940) General Theory of Elastic Shells. Prikladnaya Matamatika Mekhanika, 4, 7-34.

[32] Mcivor, I.K. (1966) Elastic Cylindrical Shell under Radial Impulse. Journal of Applied Mechanics, 33, 831-837.

[33] Mcivor, I.K. and Lovell, E.G. (1968) Dynamic Response of Finite-Length Cylindrical Shells to Nearly Uniform Radical Impulse. Aiaa Journal, 6, 2346-2351. 
[34] Mcivor, I.K. and Sonstegard, D.A. (1966) Axisymmetric Response of a Closed Spherical Shell to a Nearly Uniform Radial Impulse. Journal of Applied Mechanics, 40, 1540-1547.

[35] Mclachilan, N.W. (1947) Theory and Application of Mathieu Functions, Oxford, Clarendon Press.

[36] Moussaoui, F. and Benamar, R. (2002) Non-Linear Vibrations of Shell-Type Structures: A Review with Bibliography. Journal of Sound and Vibration, 255, 161-184.

[37] Nayfeh, A.H. (2000) Nonlinear Interactions, New York, J. Wiley \& Sons.

[38] Nayfeh, A.H. and Arafat, H.N. (2006) Axisymmetric Vibrations of Closed Spherical Shells: Equations of Motion and Bifurcation Analysis. Structural Control \& Health Monitoring, 13, $388-416$.

[39] Nayfeh, A.H. and Raouf, R.A. (1987) Non-Linear Oscillation of Circular Cylindrical-Shells. International Journal of Solids and Structures, 23, 1625-1638.

[40] Novozhilov, V.V. (1964) The Theory of Thin Elastic Shells. P. Noordhoff Lts, Groningen, The Nethelands.

[41] Pellicano, F., Amabili, M. and Vakakis, A.F. (2000) Nonlinear Vibrations and Multiple Resonances of Fluid-Filled, Circular Shells. Part 2: Perturbation Analysis. Journal of Vibration and Acoustics-Transactions of the Asme, 122, 355-364.

[42] Qatu, M.S. (1992) Review of Shallow Shell Vibration Research. Shock Vibration Digest, 24, 3-15.

[43] Rappaz, M., Bellet, M. and Deville, M. (2003) Numerical Modelling in Materials Science and Engineering, New York, Springer-Verlag.

[44] Reddy, J.N. (1984a) Exact-Solutions of Moderately Thick Laminated Shells. Journal of Engineering Mechanics-Asce, 110, 794-809.

[45] Reddy, J.N. (1984b) A Refined Nonlinear-Theory of Plates with Transverse-Shear Deformation. International Journal of Solids and Structures, 20, 881-896.

[46] Reddy, J.N. and Chandrashekhara, K. (1985a) Geometrically Non-Linear Transient Analysis of Laminated, Doubly Curved Shells. International Journal of Non-Linear Mechanics, 20, 79-90.

[47] Reddy, J.N. and Chandrashekhara, K. (1985b) Nonlinear-Analysis of Laminated Shells Including Transverse-Shear Strains. Aiaa Journal, 23, 440-441.

[48] Reddy, J.N. and Liu, C.F. (1985) A Higher-Order Shear Deformation-Theory of Laminated Elastic Shells. International Journal of Engineering Science, 23, 319-330. 
[49] Reissner, E. (1955a) Nonlinear Effects in Vibrations of Cylindrical Shells, Aeromechanics Report No. Am 5-6. Guided Missle Research Division, The Ramo-Wooldridge Corp.

[50] Reissner, E. (1955b) On Transverse Vibration of Thin Shallow Shells. J.Q. Appl. Math, 13, 169-180.

[51] Sanders, J.L. (1959) An Improved First Approximation Theory for Thin Shells. NASA $T R-R 24$.

[52] Shi, M.X., Li, Q.M. and Huang, Y. (2009a) Internal Resonance of Vibrational Modes in Single-Walled Carbon Nanotubes. Proceedings of the Royal Society a-Mathematical Physical and Engineering Sciences, 465, 3069-3082.

[53] Shi, M.X., Li, Q.M. and Huang, Y. (2009b) A Nonlocal Shell Model for Mode Transformation in Single-Walled Carbon Nanotubes. Journal of Physics-Condensed Matter, 21, 455301.

[54] Shi, M.X., Li, Q.M., Liu, B., Feng, X.Q. and Huang, Y. (2009c) Atomic-Scale Finite Element Analysis of Vibration Mode Transformation in Carbon Nanorings and Single-Walled Carbon Nanotubes. International Journal of Solids and Structures, 46, 4342-4360.

[55] Struble, R.A. (1962) Nonlinear Differential Equations, New York, McGraw-Hill Book Company, Inc.

[56] Thomas, O., Touze, C. and Chaigne, A. (2005) Non-Linear Vibrations of Free-Edge Thin Spherical Shells: Modal Interaction Rules and 1: 1:2 Internal Resonance. International Journal of Solids and Structures, 42, 3339-3373.

[57] Thomas, O., Touzé, C. and Luminais, E. (2007) Non-Linear Vibration of Free-Edge Thin Spherical Shells: Experiments on a 1:1:2 Internal Resonance. Nonlinear Dynamics, 49, 259-284.

[58] Touze, C. and Thomas, O. (2006) Non-Linear Behaviour of Free-Edge Shallow Spherical Shells: Effect of the Geometry. International Journal of Non-Linear Mechanics, 41, 678-692.

[59] Yamaki, N. (1984) Elastic Stability of Circular Cylindrical Shells, Amsterdam, North_holland.

[60] Ye, Z.M. (1997) The Non-Linear Vibration and Dynamic Instability of Thin Shallow Shells. Journal of Sound and Vibration, 202, 303-311.

[61] Zhu, W.H., Xue, H.L., Zhou, G.Q. and Schleyer, G.K. (1997) Dynamic Response of Cylindrical Explosive Chambers to Internal Blast Loading Produced by a Concentrated Charge. International Journal of Impact Engineering, 19, 831-845. 


\section{Figures:}

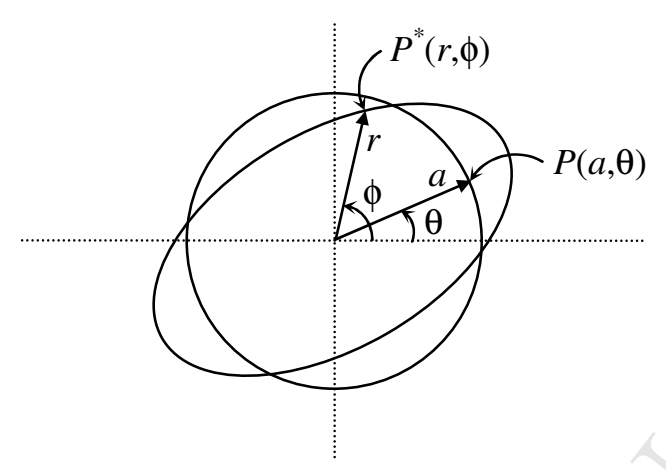

Fig.2.1 A schematic representation of the deformation of a cylindrical shell with centroid fixed.

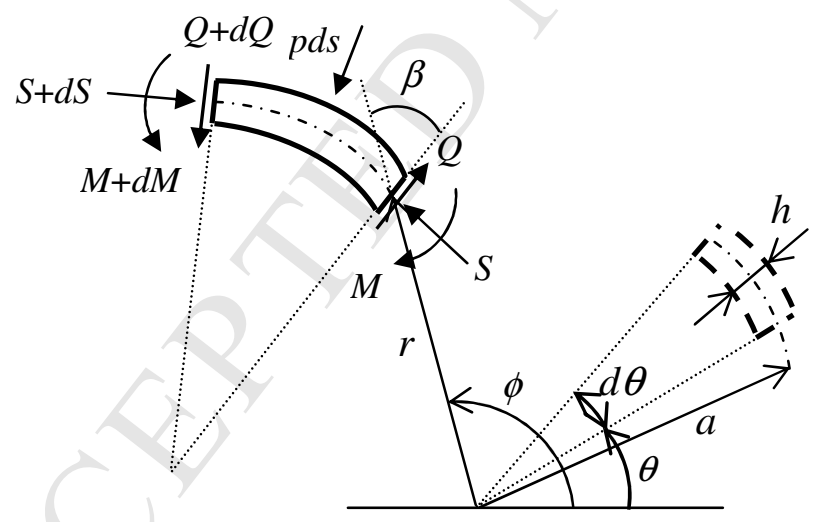

Fig.2.2 A typical cylindrical shell element: undeformed element (right) and deformed element (left). 


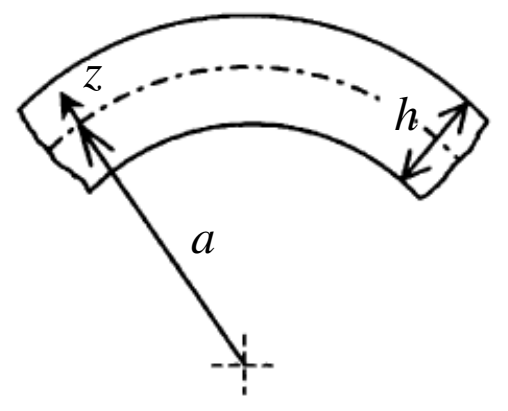

Fig.2.3 Part of cross-section of circular cylindrical shell with a representative point shown at distance $\mathrm{z}$ above middle surface.

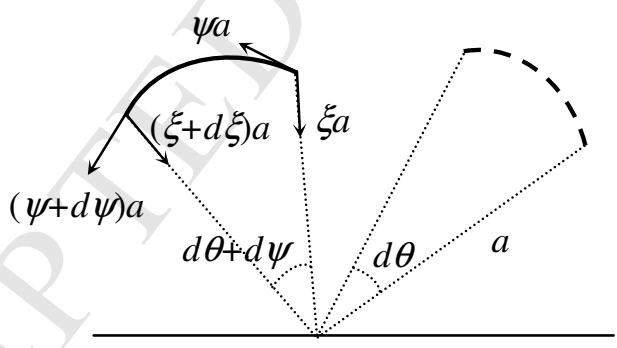

Fig. 2.4 A schematic representation showing initial state (dash element) to current configuration (solid element) for a typical middle surface element. Arrows on both ends show the radial and tangential displacements, respectively. 

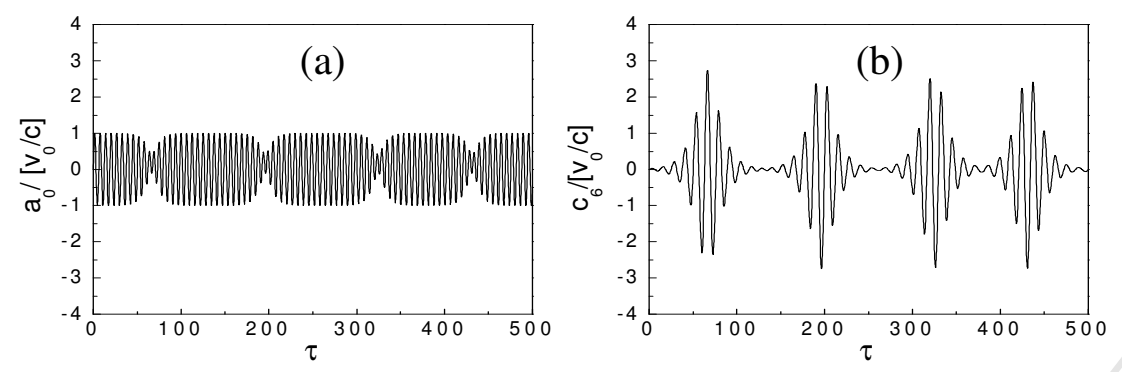

Fig.3.1 Coupled motion of a cylindrical shell within Goodier and McIvor's model $(a / h=20.4$, $v_{0} / c=0.005$ ). (a) RBM $a_{0}$, (b) Inextensional flexural mode $c_{6}$.
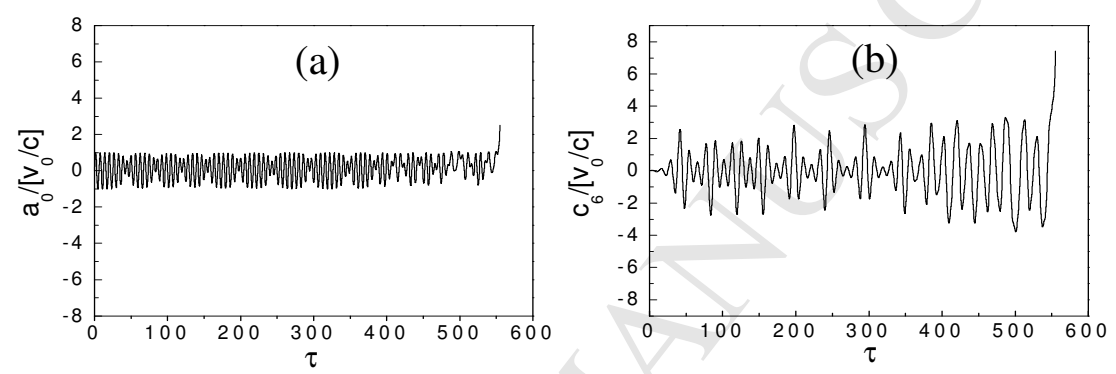

Fig.3.2 Coupled motion of a cylindrical shell within Goodier and McIvor's model $(a / h=20.4$, $v_{0} / c=0.0075$ ). (a) RBM $a_{0}$, (b) Inextensional flexural mode $c_{6}$.
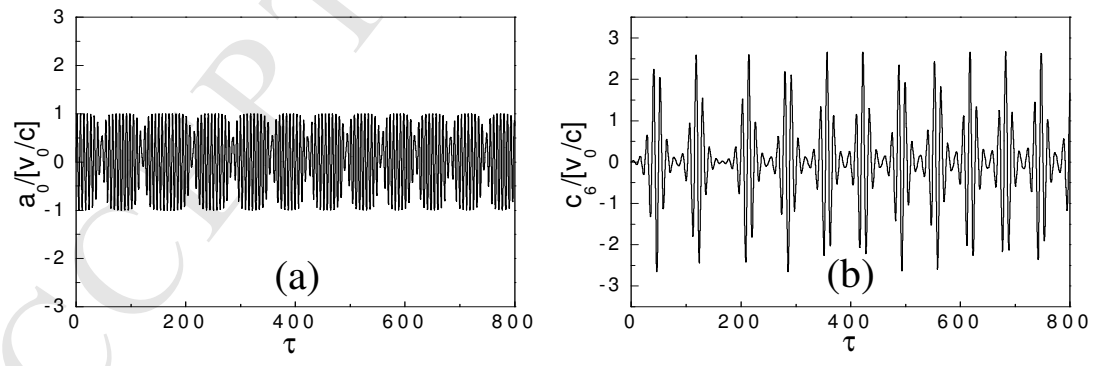

Fig.3.3 Coupled motion of a cylindrical shell within Goodier and McIvor's model but with the fourth-order term included $\left(a / h=20.4, v_{0} / c=0.0075\right)$. (a) $\mathrm{RBM} a_{0}$, (b) Inextensional flexural mode $c_{6}$. 

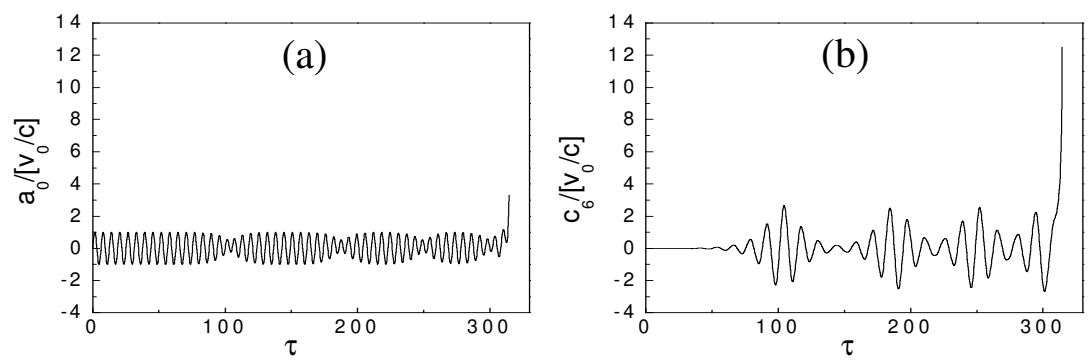

Fig.3.4 Coupled motion of a cylindrical shell within McIvor's model $\left(a / h=20.4, v_{0} / c=0.005\right)$.

(a) RBM $a_{0}$, (b) Inextensional flexural mode $c_{6}$.
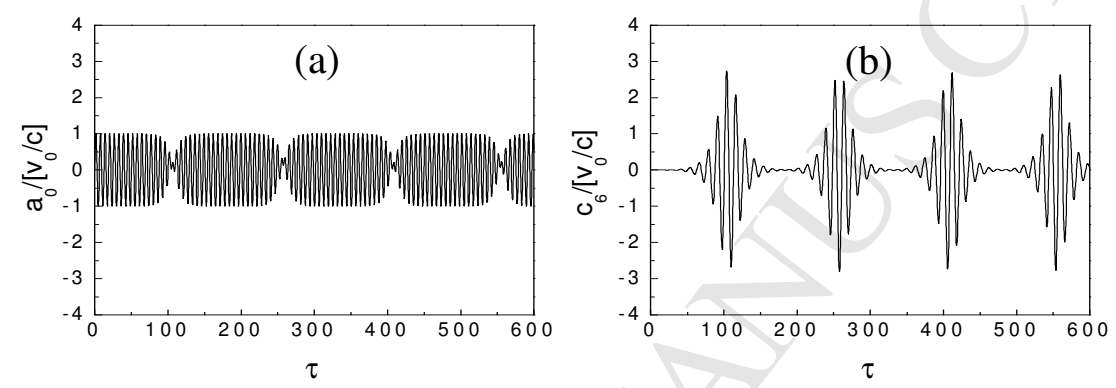

Fig.3.5 Coupled motion of a cylindrical shell within present explicit model $(a / h=20.4$, $v_{0} / c=0.005$ ). (a) RBM $a_{0}$, (b) Inextensional flexural mode $c_{6}$.
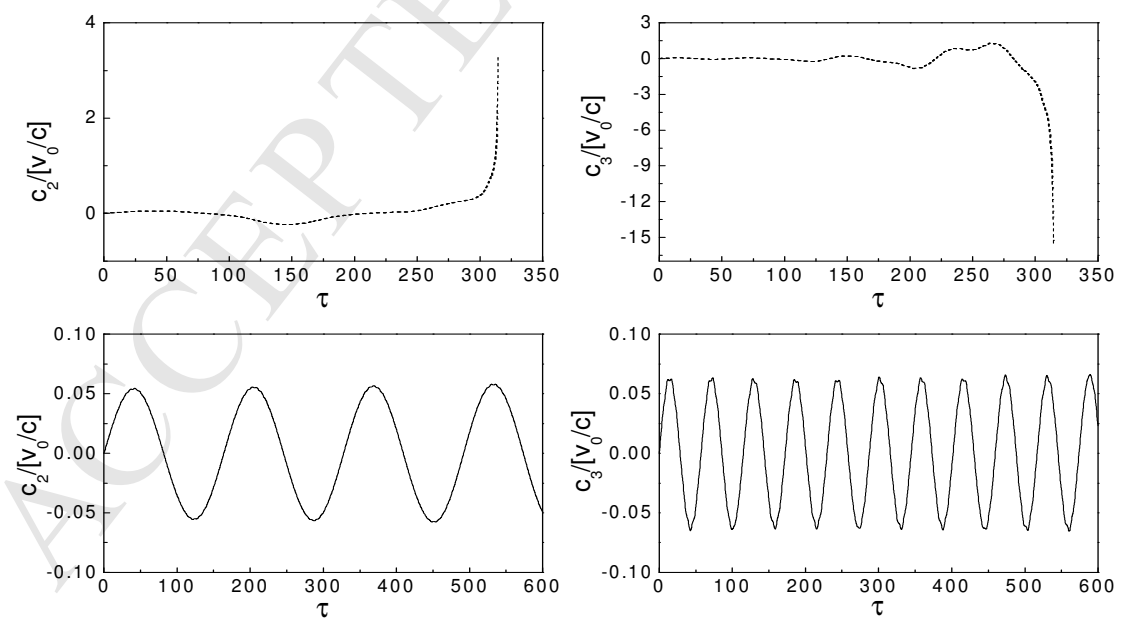

Fig.3.6 Vibration histories of the two lowest flexural modes $c_{2}$ and $c_{3}$ of a cylindrical shell $\left(a / h=20.4, v_{0} / c=0.005\right)$. Solid and dashed lines correspond to present explicit model and McIvor's explicit model, respectively. 


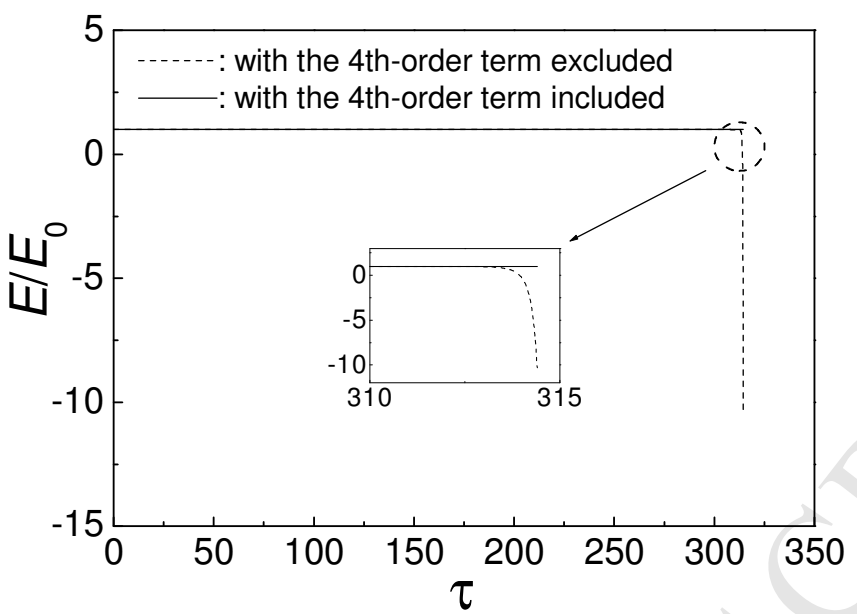

Fig.3.7 Energy history of a cylindrical shell $\left(a / h=20.4, v_{0} / c=0.005\right)$ with and without the fourth-order term included in strain energy. $E_{0}$ is the initial total energy.

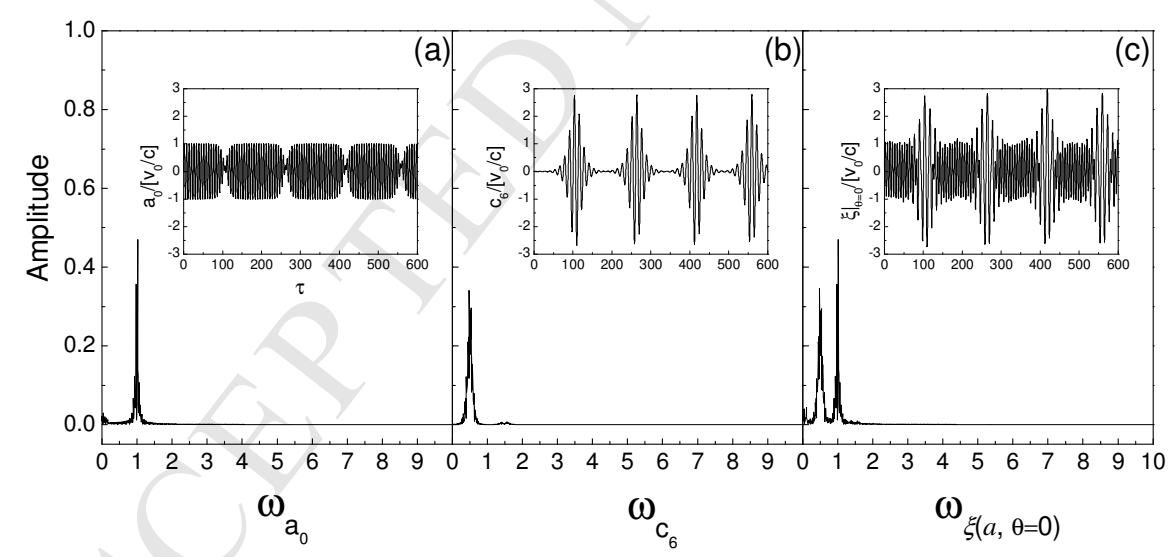

Fig.3.8 Response in frequency domain at a selected point of a cylindrical shell $(a / h=20.4$, $v_{0} / c=0.005$ ).(a) $\operatorname{RBM} a_{0}$, (b) Inextensional flexural mode $c_{6}$, (c) the total response $\xi(a, \theta=0)$ 

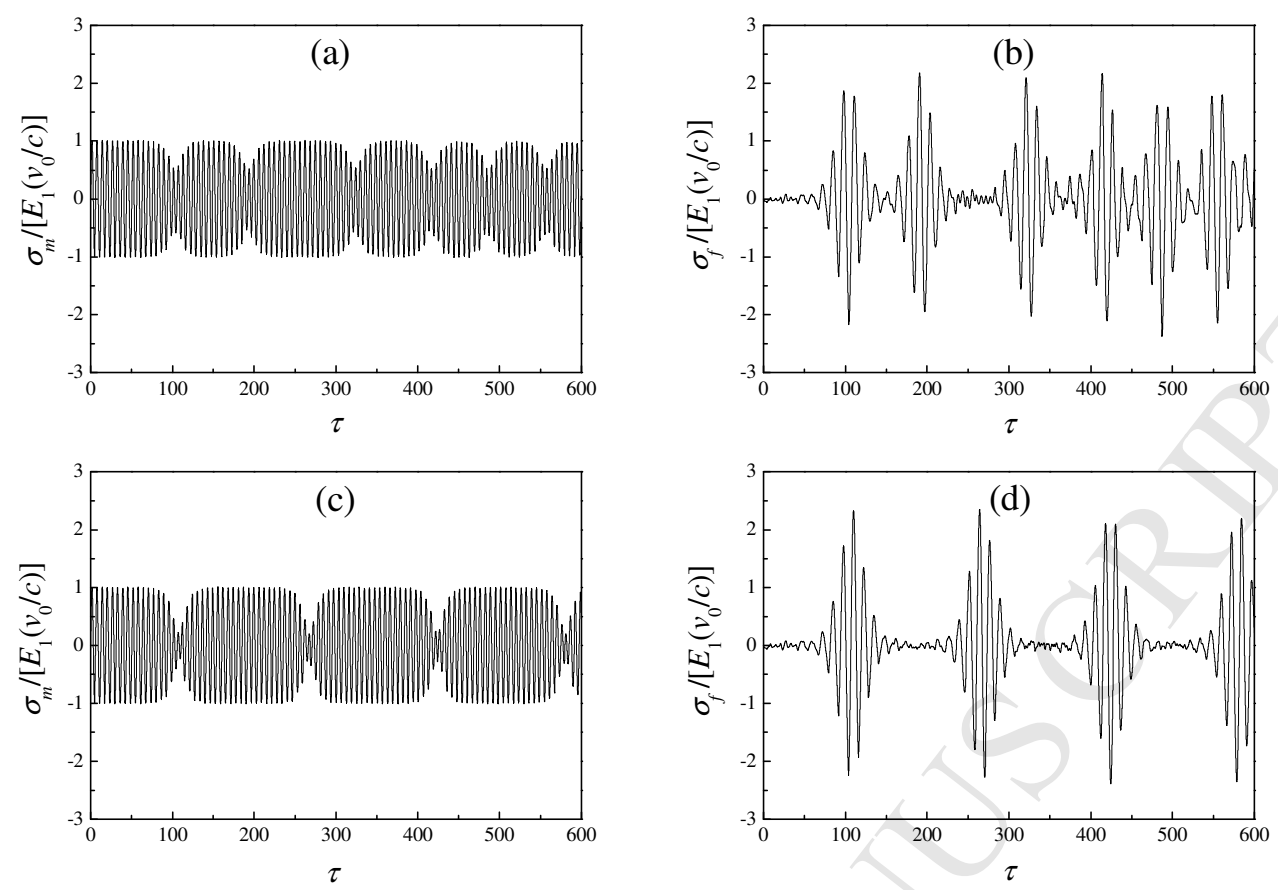

Fig.4.1 (a) and (b), (c) and (d) are the membrane and flexural stresses of a cylindrical shell based respectively on the second- and third-order implicit model $\left(a / h=20.4, v_{0} / c=0.005\right)$. 

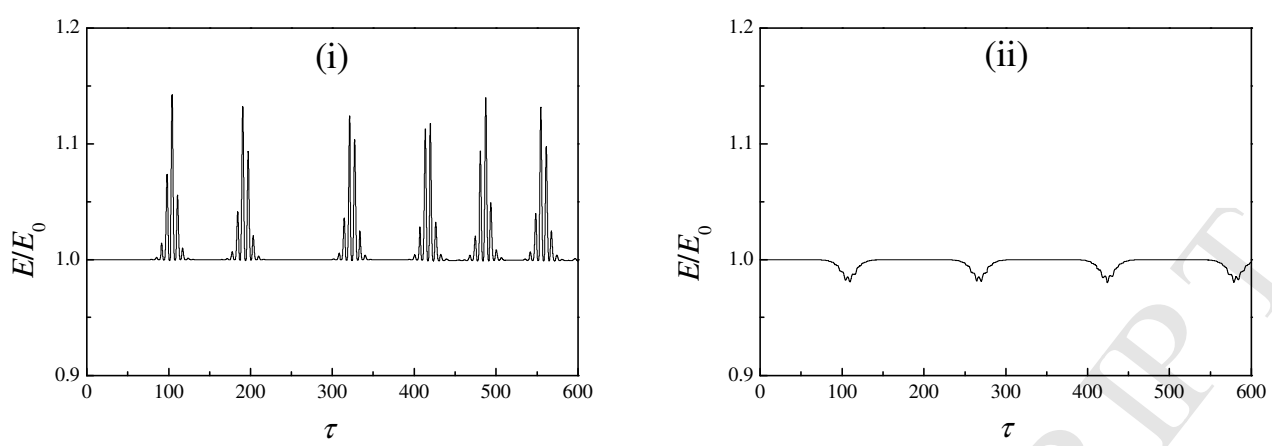

Fig.4.2 (i) and (ii) are the total energy of a cylindrical shell based respectively on the second- and third-order implicit model $\left(a / h=20.4, v_{0} / c=0.005\right)$. 

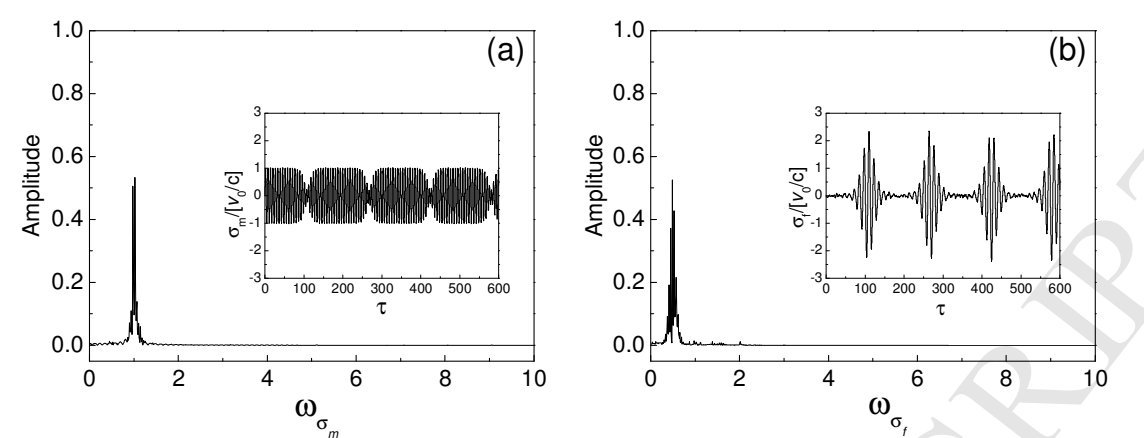

Fig.4.3 (a) and (b) are the frequency spectra of the membrane and flexural stresses based on the third-order implicit model, respectively $\left(a / h=20.4, v_{0} / c=0.005\right)$. The inset in each of them is the stress itself correspondingly. 

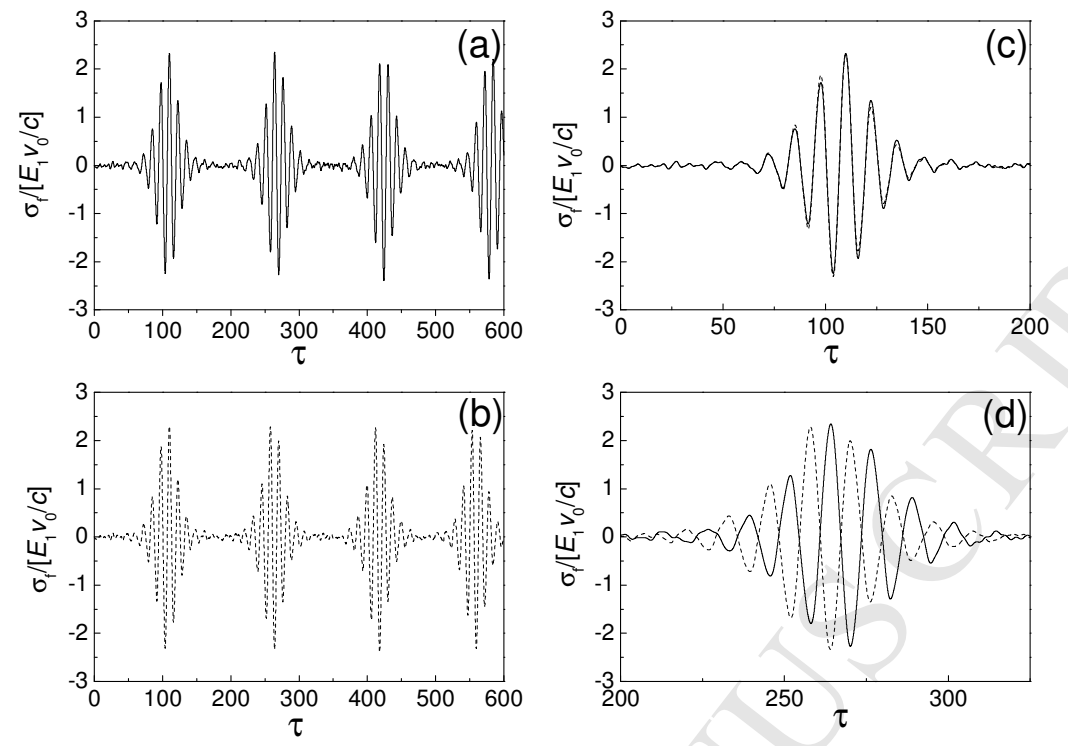

Fig.4.4 (a) and (b) are the responses of flexural stresses from the third-order implicit model (solid line) and present explicit model (dashed line), respectively. (c) and (d) are the superposed intercepts of them of the first and second group of stress peaks, respectively $(a / h=20.4$, $\left.v_{0} / c=0.005\right)$. 


\section{Figures:}

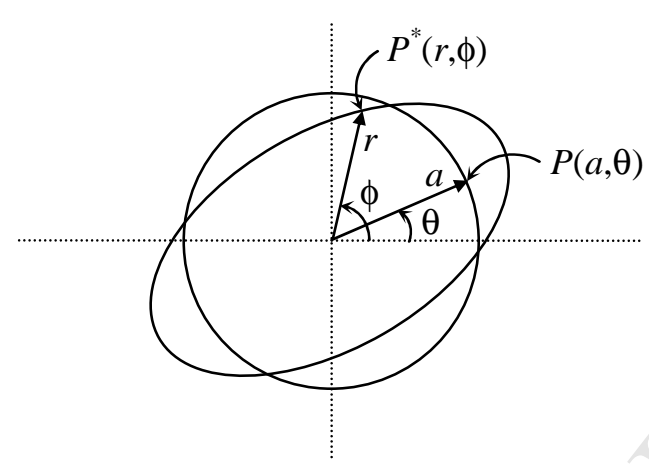

Fig.2.1 A schematic representation of the deformation of a cylindrical shell with centroid fixed.

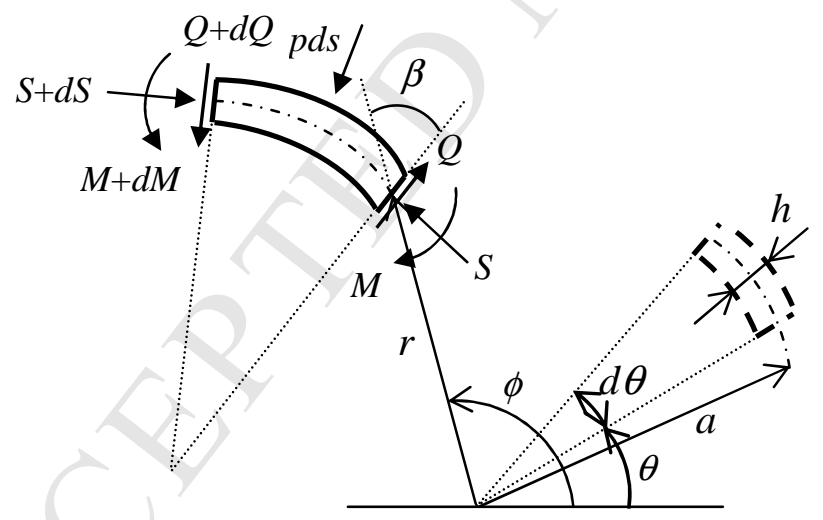

Fig.2.2 A typical cylindrical shell element: undeformed element (right) and deformed element (left). 


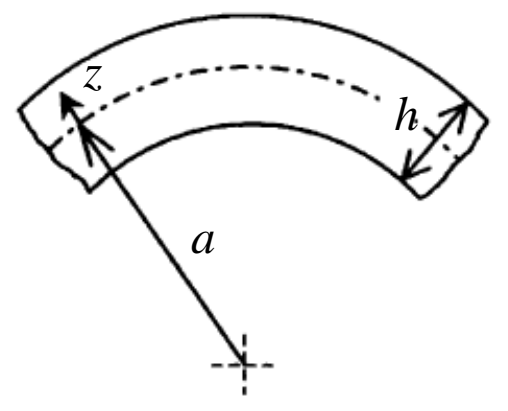

Fig.2.3 Part of cross-section of circular cylindrical shell with a representative point shown at distance $\mathrm{z}$ above middle surface.

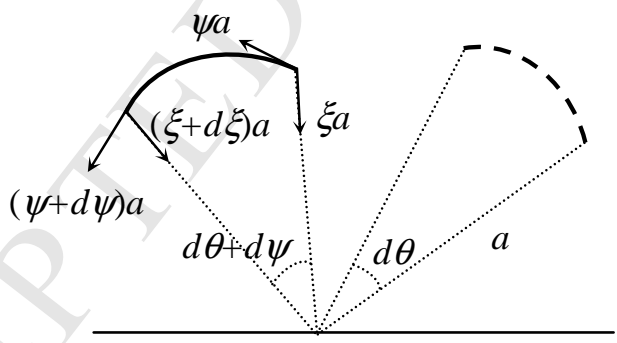

Fig. 2.4 A schematic representation showing initial state (dash element) to current configuration (solid element) for a typical middle surface element. Arrows on both ends show the radial and tangential displacements, respectively. 

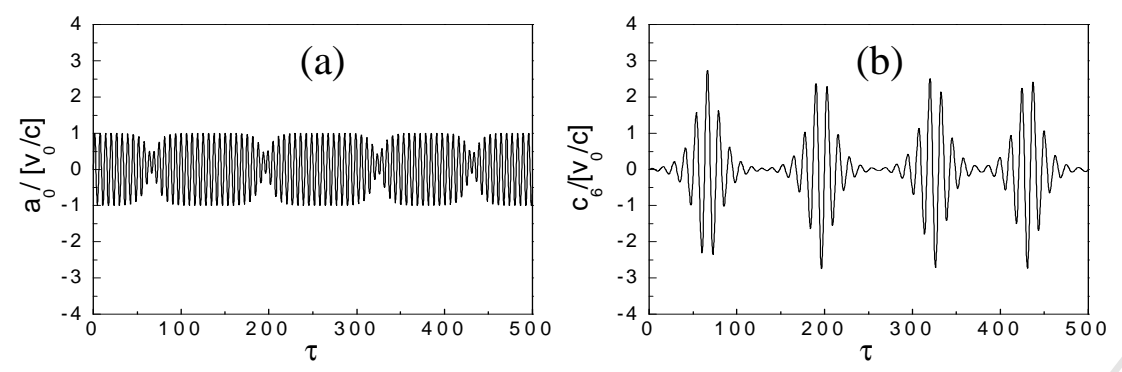

Fig.3.1 Coupled motion of a cylindrical shell within Goodier and McIvor's model $(a / h=20.4$, $v_{0} / c=0.005$ ). (a) RBM $a_{0}$, (b) Inextensional flexural mode $c_{6}$.
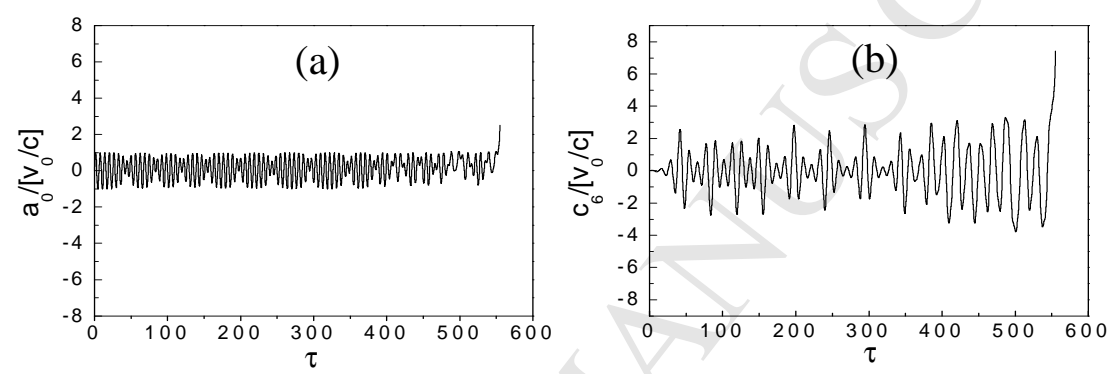

Fig.3.2 Coupled motion of a cylindrical shell within Goodier and McIvor's model $(a / h=20.4$, $v_{0} / c=0.0075$ ). (a) RBM $a_{0}$, (b) Inextensional flexural mode $c_{6}$.
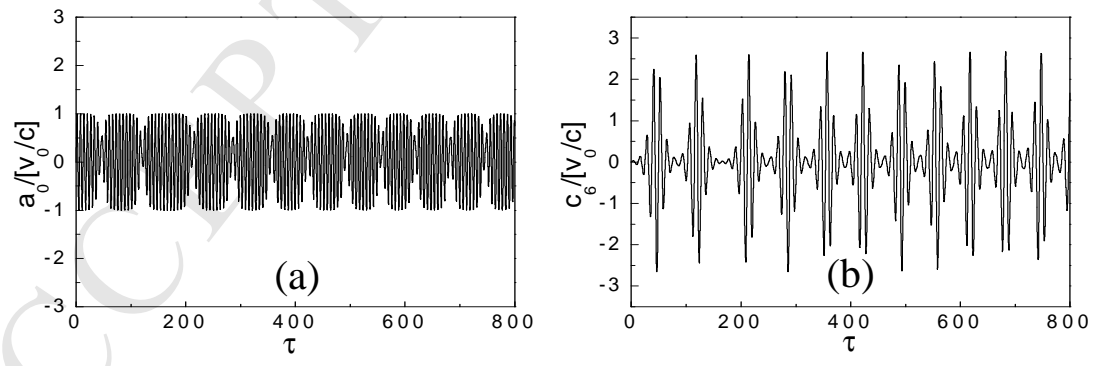

Fig.3.3 Coupled motion of a cylindrical shell within Goodier and McIvor's model but with the fourth-order term included $\left(a / h=20.4, v_{0} / c=0.0075\right)$. (a) RBM $a_{0}$, (b) Inextensional flexural mode $c_{6}$. 

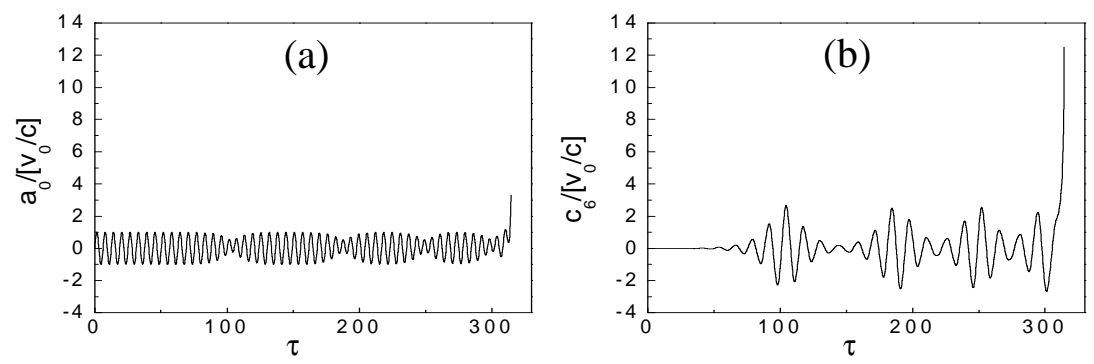

Fig.3.4 Coupled motion of a cylindrical shell within McIvor's model $\left(a / h=20.4, v_{0} / c=0.005\right)$.

(a) RBM $a_{0}$, (b) Inextensional flexural mode $c_{6}$.
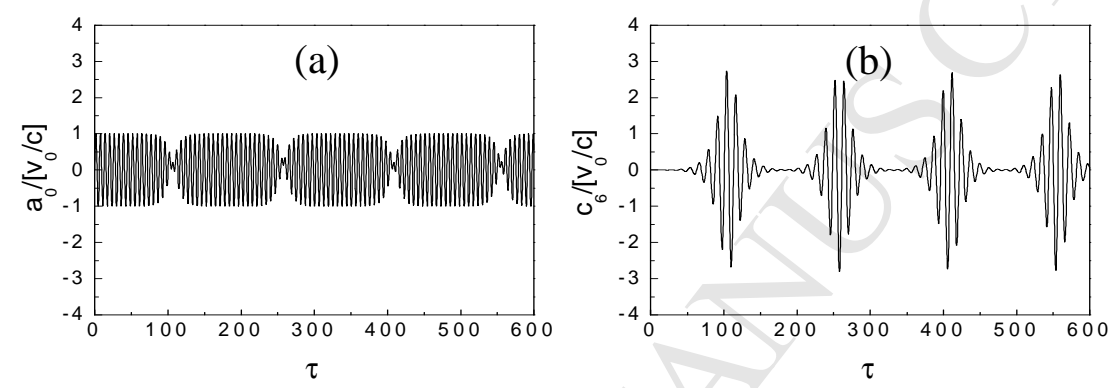

Fig.3.5 Coupled motion of a cylindrical shell within present explicit model $(a / h=20.4$, $v_{0} / c=0.005$ ). (a) RBM $a_{0}$, (b) Inextensional flexural mode $c_{6}$.
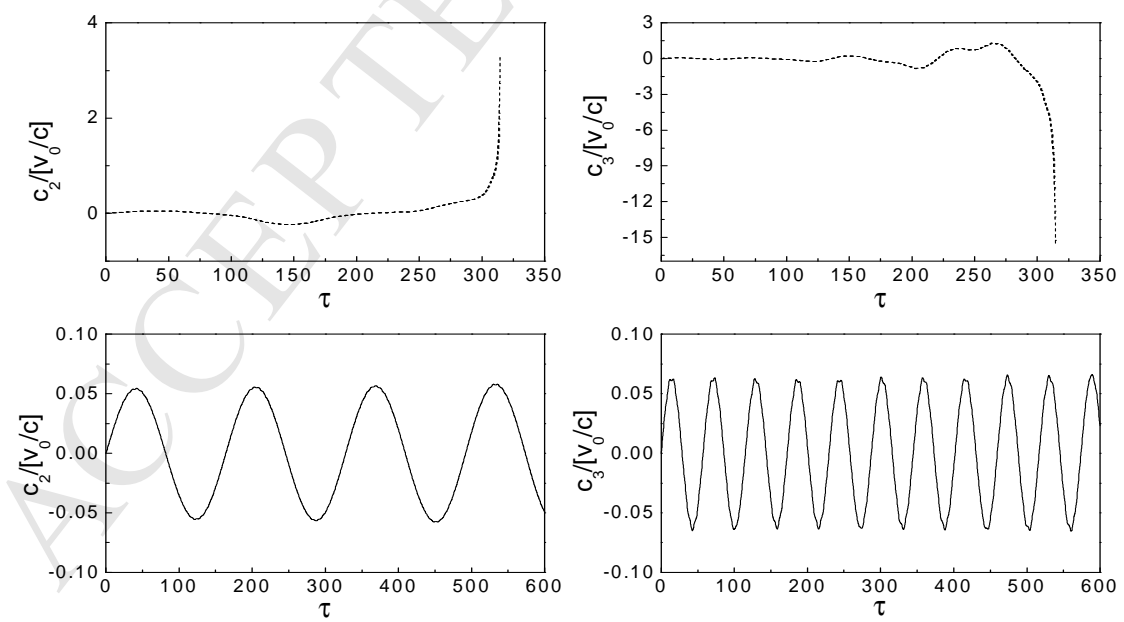

Fig.3.6 Vibration histories of the two lowest flexural modes $c_{2}$ and $c_{3}$ of a cylindrical shell $\left(a / h=20.4, v_{0} / c=0.005\right)$. Solid and dashed lines correspond to present explicit model and McIvor's explicit model, respectively. 


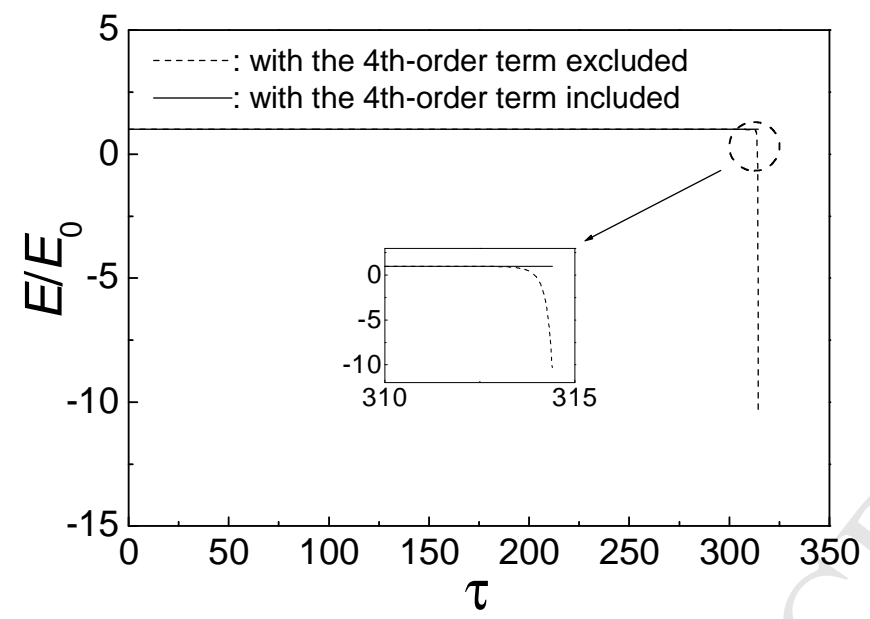

Fig.3.7 Energy history of a cylindrical shell $\left(a / h=20.4, v_{0} / c=0.005\right)$ with and without the fourth-order term included in strain energy. $E_{0}$ is the initial total energy.

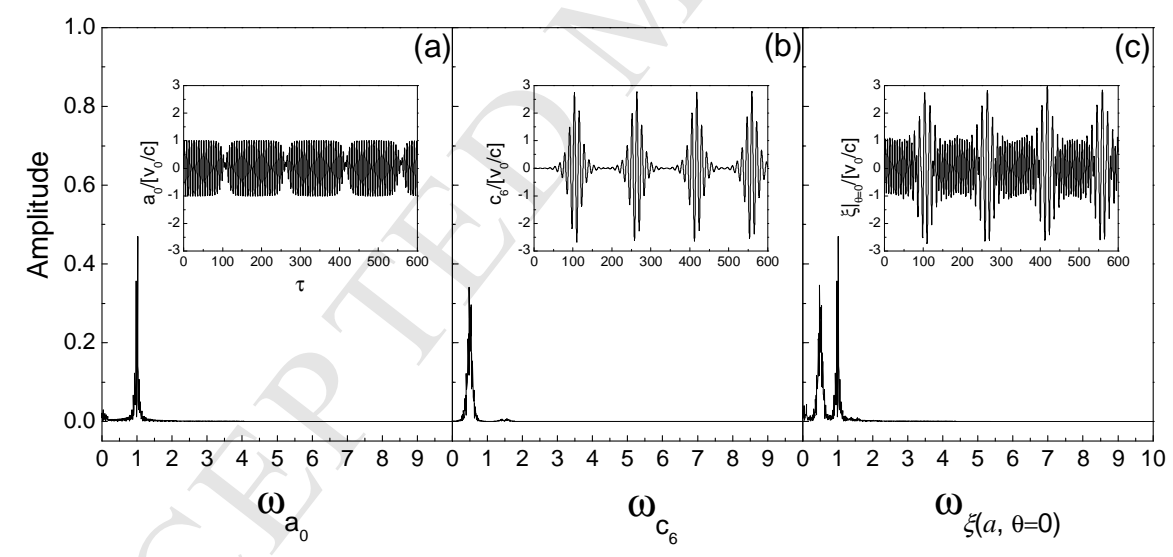

Fig.3.8 Response in frequency domain at a selected point of a cylindrical shell $(a / h=20.4$, $v_{0} / c=0.005$ ).(a) RBM $a_{0}$, (b) Inextensional flexural mode $c_{6}$, (c) the total response $\xi(a, \theta=0)$ 

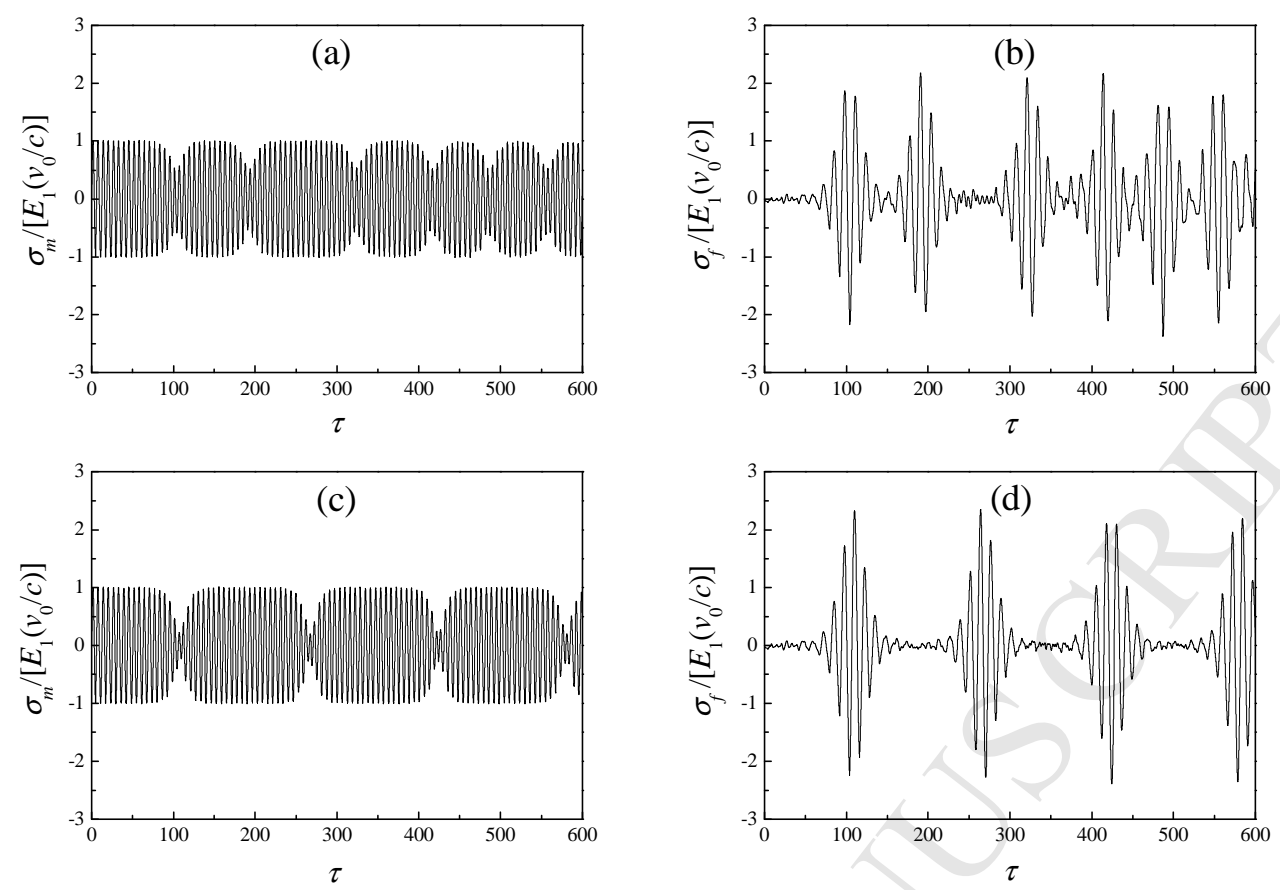

Fig.4.1 (a) and (b), (c) and (d) are the membrane and flexural stresses of a cylindrical shell based respectively on the second- and third-order implicit model $\left(a / h=20.4, v_{0} / c=0.005\right)$. 

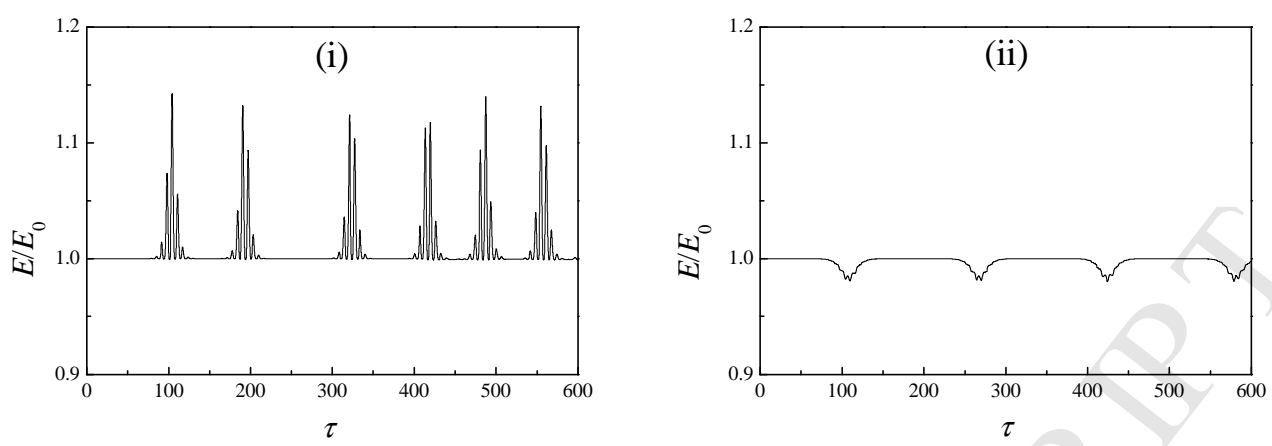

Fig.4.2 (i) and (ii) are the total energy of a cylindrical shell based respectively on the second- and third-order implicit model $\left(a / h=20.4, v_{0} / c=0.005\right)$. 

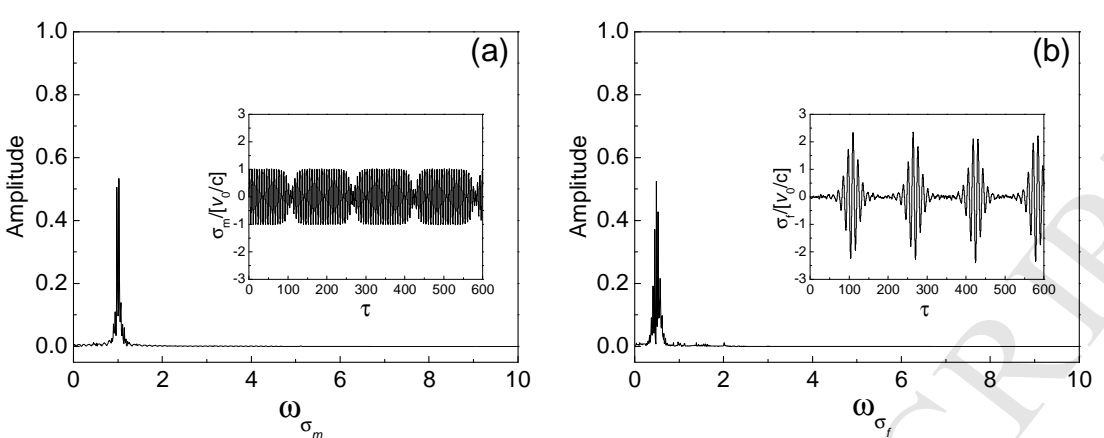

Fig.4.3 (a) and (b) are the frequency spectra of the membrane and flexural stresses based on the third-order implicit model, respectively $\left(a / h=20.4, v_{0} / c=0.005\right)$. The inset in each of them is the stress itself correspondingly. 

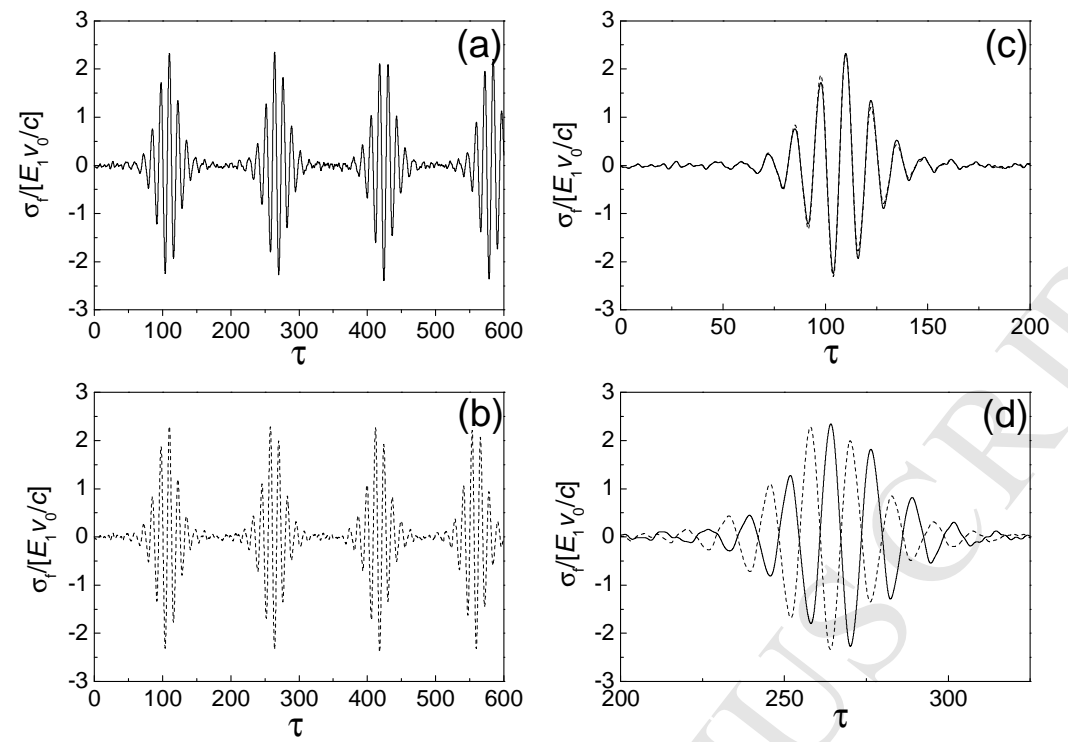

Fig.4.4 (a) and (b) are the responses of flexural stresses from the third-order implicit model (solid line) and present explicit model (dashed line), respectively. (c) and (d) are the superposed intercepts of them of the first and second group of stress peaks, respectively $(a / h=20.4$, $\left.v_{0} / c=0.005\right)$. 